\title{
Bioclimática, mediciones ambientales y uso de los espacios urbanos: Evaluación comparativa en la Plaza de Chamberí, Madrid
}

\author{
*Nagore Urrutia del Campo \\ **Javier Neila González
}

Artículo

\section{Afiliación:}

*Universidad del País Vasco, España.

**Universidad Politécnica de Madrid, España.

E-mail: nagore.urrutia@ehu.eus

fjavier.neila@upm.es

Recibido: 16 de agosto del 2019

Aceptado: 7 de noviembre del 2019

\begin{abstract}
Nagore Urrutia Del Campo
Arquitecta Superior por la Universidad del País Vasco EHU, Máster en Medio Ambiente y Arquitectura Bioclimática y Máster Oficial e Periferias, Sostenibilidad y Vitalidad Urbana por la Universidad Politécnica de Madrid UPM. Profesora e investigadora del grupo CAVIAR-EHU. Arquitecta independiente.
\end{abstract}

\section{Francisco Javier Neila González}

Arquitecto Superior. Catedrático en la Universidad Politécnica de Madrid UPM. Director del Grupo de Investigación ABIO-UPM. Director del Máster Medio Ambiente y Arquitectura Bioclimática.

\section{Resumen}

Este trabajo de investigación se centra en el estudio teórico y experimental del microclima urbano de la plaza Chamberí de Madrid, ciudad con una marcada Isla de Calor. Para ello, se realizaron mediciones in-situ de temperatura superficial de materiales en fachadas, pavimentos y mobiliario urbano, de temperatura seca, humedad relativa del aire y de velocidad y dirección del viento en condiciones de soleamiento y sombreamiento. Se levantó el entorno, la vegetación, arbolado y materiales de acabado en 3D para desarrollar simulaciones de soleamiento y radiación solar. Paralelamente, se registró el uso que las personas hacían del espacio urbano. Finalmente se compararon los resultados con un estudio bioclimático tradicional que serviría para definir el confort. Se concluyó que las herramientas bioclimáticas son efectivas para el diseño de espacios confortables térmicamente, siempre y cuando se creen microclimas alternativos que den opción de adaptarse al entorno. Las mediciones de campo revelan la importancia de las propiedades térmicas de los materiales y la necesidad de realizar estudios de soleamiento para la selección y localización de materiales con diversas propiedades higrotérmicas y el correcto empleo de suelos naturales, vegetación y agua. Se observa que las estrategias teóricas para alcanzar el confort definidas mediante las herramientas tradicionales, en numerosas ocasiones, no coincide con el uso real del espacio público. Además, se detectaron notables diferencias entre los datos climáticos históricos, los de la estación meteorológica estatal más cercana y las mediciones locales. Se detecta numéricamente el efecto de Isla de Calor Urbana en la plaza, respecto al parque del Retiro.

Palabras clave: confort; microclima; diseño urbano; uso del espacio; Isla de Calor Urbana.

Bioclimatic architecture, environmental measures, and urban spaces usage: Comparative evaluation in Plaza de Chamberí, Madrid

\begin{abstract}
This theoretical and applied research analyses the microclimate of Chamberí Square in Madrid, a city with an intense Urban Heat Island effect. Contact temperature of finishing materials of facades, pavements, and urban furniture were measured. Dry air temperature, relative humidity, wind velocity and direction were measured too, both in the sun and in the shade. Shading and solar radiation simulations were carried out by constructing a 3D model defining finishing materials' properties, vegetation, and existing trees. Public space users were also located, and their activities registered. The obtained data was compared to a traditional bioclimatic study. It is concluded that these studies are effective to design comfortable urban spaces because they are useful in order to create alternative microclimates. Field measurements reveal essential sun radiation and shading studies in urban design for a proper selection and location of materials with a variety of thermal properties and for the correct use of natural soils, vegetation, and water. The comfort strategies defined by traditional bioclimatic tools and the real use of urban space do not come together. Noticeable differences between historical climate data, estate weather station, and local measurements are detected. The Urban Heat Island of the square is numerically defined.
\end{abstract}

Keywords: comfort; outdoor microclimate; urban design; outdoor activities; Urban Heat Island. 


\section{Introducción}

os cambios atmosféricos relacionados con la urbanización comenzaron a analizarse en el siglo XIX (Howard, 1883), aunque la técnica bioclimática y la investigación en torno al microclima y su relación con el diseño urbano tomaron importancia hace más de 50 años (Givoni, 1998; Olgyay, 1999). A mediados del siglo XX con la aparición de los sistemas de acondicionamiento activos. Tanto la arquitectura como el urbanismo se desvincularon de su relación con el clima, la búsqueda del bienestar higrotérmico y optimización energética mediante el propio diseño.

La influencia en la modificación del microclima urbano tanto de los diversos elementos ambientales como de las propiedades de los materiales urbanos o la vegetación, entre otros, ha sido analizada a lo largo de los últimos 20 años (RUROS Project, 2004; Erell, Pearlmutter \& Williamson, 2010). Más recientemente, los estudios sobre el microclima urbano se han centrado en la Isla de Calor Urbana (ICU) superficial, atmosférica y cambio climático (Oke, 1973; Landsberg, 1981; Owen, Carlson \& Gillies , 1998) en su mitigación (Brown, 2011) y en sus efectos en la salud pública (Coutts \& Harris, 2013). Teniendo en cuenta las cada vez más frecuentes y profundas olas de calor (IPCC, 2007; Luber \& McGeehin, 2008), las estrategias de adaptación al cambio climático para la mejora del confort higrotérmico en las ciudades suponen un reto para la mejora de la calidad de vida, como lo es evitar enfermedades y reducir la mortalidad ligada a los golpes de calor (European Commission, 2017; Smargiassi et al., 2009).

Existen numerosas variables y estrategias de regulación del microclima urbano ligadas al diseño urbano: el empleo del agua, los materiales de acabado, la vegetación, el sombreamiento o la creación de microbrisas, entre otras. Con base a esas condiciones ambientales, el confort en los espacios abiertos se verá condicionada (Shooshtarian, Rajagopalan \& Sagoo, 2018). Con una población urbana que supera a la población rural (Population Reference Bureau, 2009) y en un escenario de cambio climático, el confort en espacios exteriores y la creación de espacios abiertos de calidad ha sido ampliamente analizado. Éste está condicionado por variables ergonómicas, psicológicas, de usos y fisiológicas. Entre éstas últimas se encuentra el confort higrotérmico. La relación entre el confort higrotérmico y los parámetros de comportamiento ha sido analizada en las últimas tres décadas (Chen \& Ng, 2012) y se han creado numerosos índices de medición del mismo como el Predicted Mean Vote Index (PMV) (Fanger, 1982), Physiological Equivalent Temperature (PET) (Mayer \& Höppe, 1987), Thermal Sensation Vote TS (Givoni, et al., 2003) o Actual Sensation Vote ASV (Nikolopoulou \& Lykoudis, 2006), entre otros. Existen, además, numerosos estudios que relacionan los resultados derivados de estos índices de confort (Predicted Percentage Dissatisfied PPD) y su relación con el confort percibido (Actual Sensation Vote ASV) o el uso que se realiza de los espacios urbanos, existiendo una gran discrepancia en los resultados obtenidos (Pearlmutter, Jiao \& Garb, 2014; Lenzholzer, 2010).

En este estudio, se selecciona un espacio abierto de Madrid, ciudad de condiciones climáticas muy variables y extremas que condicionan la utilización de los espacios exteriores, con los siguientes objetivos:

a. Determinar el comportamiento microclimático del espacio mediante mediciones in-situ y el uso que hacen las personas del mismo.

b. Identificar las relaciones entre estudios climáticos teóricos y las acciones de adaptación al entorno climático que llevan a cabo las personas.

\section{Contexto geográfico}

La ciudad de Madrid en España tiene un clima templado con veranos secos y calientes, según la clasificación de Köppen Hagen Csa. Es un clima con variables climáticas muy diferenciadas entre el invierno y el verano, acusados contrastes de temperatura de hasta casi $30^{\circ} \mathrm{C}$ entre invierno y verano y diferencias día-noche de su temperatura media de hasta $17^{\circ} \mathrm{C}$ en agosto y $7^{\circ} \mathrm{C}$ en invierno. Hay numerosos meses infracalentados, pero desde mediados de mayo hasta mediados de octubre se alcanzarán temperaturas que 
superarán las temperaturas de confort. Los meses de mayor radiación global horizontal coinciden con aquellos de mayores temperaturas. La temperatura media máxima es de $31,16^{\circ} \mathrm{C}$ en julio, con una irradiancia global horizontal de $860 \mathrm{Wm} 2 / \mathrm{h}$.

El clima es seco, con una humedad relativa media baja, alcanza el mínimo del $39 \%$ en julio y el máximo de 74\% en diciembre. En las horas de más uso del espacio público (8:00 a.m.-10:00 p.m.) la mayor parte del año la humedad relativa se encuentra por debajo del $40 \%$ y en verano incluso por debajo del $20 \%$. La temperatura media en Madrid ha tenido una tendencia al alza en los últimos 135 años (1881-2015) de aproximadamente $1,5^{\circ} \mathrm{C}$, tanto en sus valores medios anuales como en los mensuales. Este incremento se hace especialmente notable a partir de 1950.

\section{Temperatura media anual}

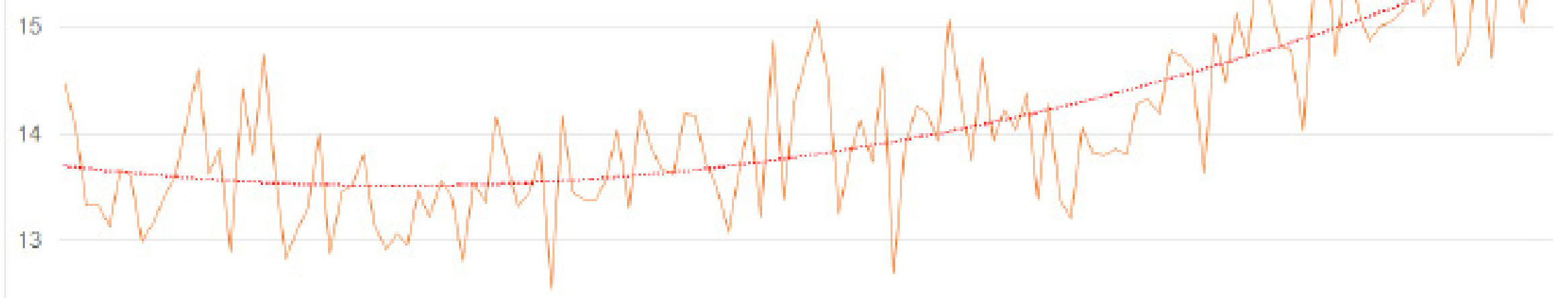

12

11

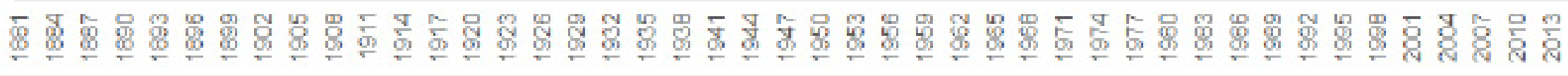

Figura 1. Temperaturas medias anuales de Madrid, periodo 1881-2015.

Elaboración a partir de datos históricos de la Agencia Estatal de Meteorología AEMET.
Madrid se localiza en el sur de Europa en el interior peninsular español, donde los efectos del cambio climático serán muy acusados (European Environment Agency, 2012). Es, además, la segunda ciudad europea, tras La Valetta, especialmente vulnerable a las olas de calor extremas (>90 C) (Smid, Russo, Costa, Granell \& Pebesma, 2019) por su tamaño, numerosas superficies artificiales, alto porcentaje de población de riesgo y sus condiciones climáticas regionales. Tiene un clima urbano muy definido con un elevado número de días con islas de calor muy intensas (Fernández, Allende, Rasilla, Martilli \& Alcaide, 2016). La ICU de Madrid no ha cambiado de intensidad desde 1988, pero se ha ampliado en superficie con una máxima diferencia de temperatura entre el centro y la periferia de entre $5^{\circ} \mathrm{C}$ y $6^{\circ} \mathrm{C}$ (Núñez, Sánchez-Guevara \& Neila, 2017).

\section{Metodología}

Esta investigación realiza un análisis multicriterio de condiciones ambientales y de la utilización que hacen las personas de la plaza, contrastando análisis teóricos con recolección de datos in-situ. A partir de la construcción del marco teórico de esta investigación se llevan a cabo los siguientes trabajos:

\section{Recopilación de datos y estudios previos:}

Los estudios previos se basan en trabajos de gabinete y trabajos de campo:

a. Caracterización física, identificación de usos y funciones: en cuanto a los aspectos físicos, se estudian la densidad edificatoria, forma, dimensiones y superficies, proporciones alto-ancho, mobiliario urbano, las calles adyacentes, hitos urbanos, pendientes, niveles, transición público-privada, mantenimiento, seguridad, accesibilidad 
y materiales de acabado. Respecto a usos y equipamientos se identifican los usos de los edificios y equipamientos privados (viviendas, iglesias, servicios sociales, tienda o restaurantes), el uso del espacio (carretera, parking, zonas peatonales, lugares estanciales, zonas de juego, zonas verdes, terrazas privadas,...), mobiliario urbano (fuentes, bancos, kiosko), hitos y referencias urbanas (monumentos) y las actividades urbanas (espontáneas o guiadas).

b. Estudio climático y microclimático. Por una parte, se han realizado los correspondientes climogramas de Olgyay (Olgyay, 1963) y de Bienestar Adaptado (Neila, 2004), determinando las estrategias para alcanzar el confort durante los meses de abril, mayo y junio. Se han diferenciado 3 actividades metabólicas diferentes: persona caminando o niño jugando $(2,95 \mathrm{met})$, persona de pie $(1,65 \mathrm{met})$ y persona sentada $(1,15 \mathrm{met})$, así como el arropamiento, que para los meses de abril y mayo será de $1 \mathrm{clo}$, mientras que para junio se reduce a 0,7 clo.
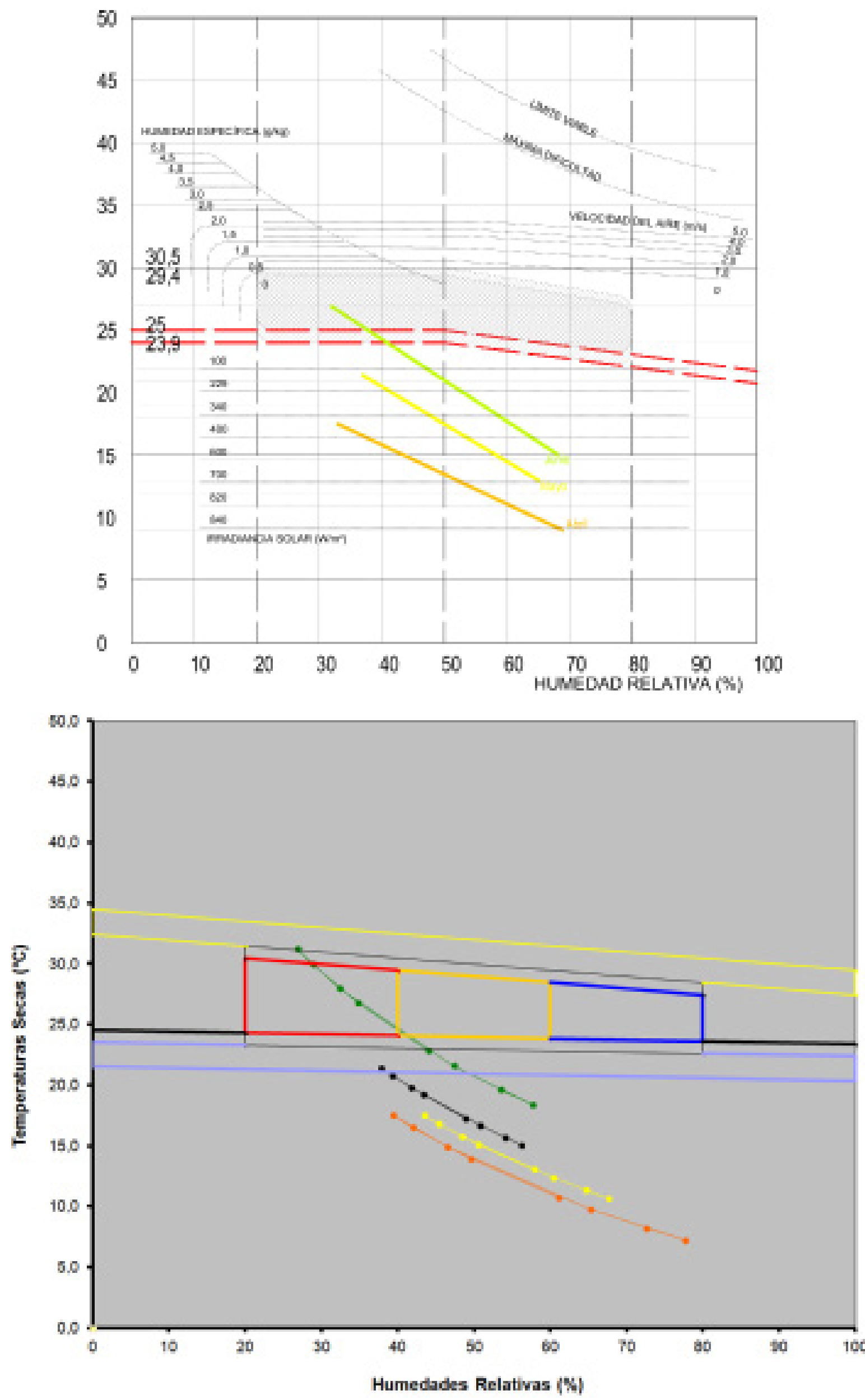

Figura 2. Climograma de Olgyay para arropamientos de 0,7 y 1 clo y CBA para arropamiento de 1 clo y actividad metabólica 1,65met, de abril a julio.

Fuente: Elaboración propia.
Para la caracterización de los diversos puntos de medición en lo referente a su disponibilidad de sombra y radiación solar, se realizó un levantamiento 3D del entorno urbano, definiendo las propiedades de sus materiales de acabado y cielo, porcentaje 
de sombra y de radiación solar en el periodo, analizado mediante el software Autodesk Ecotect Analysis (Oregi, Roth, Alsema, van Ginkel \& Struik, 2015). Se seleccionaron los puntos de medida con base a la mayor o menor ganancia solar en suelos y fachadas, los materiales de acabado y la localización de las zonas estanciales.

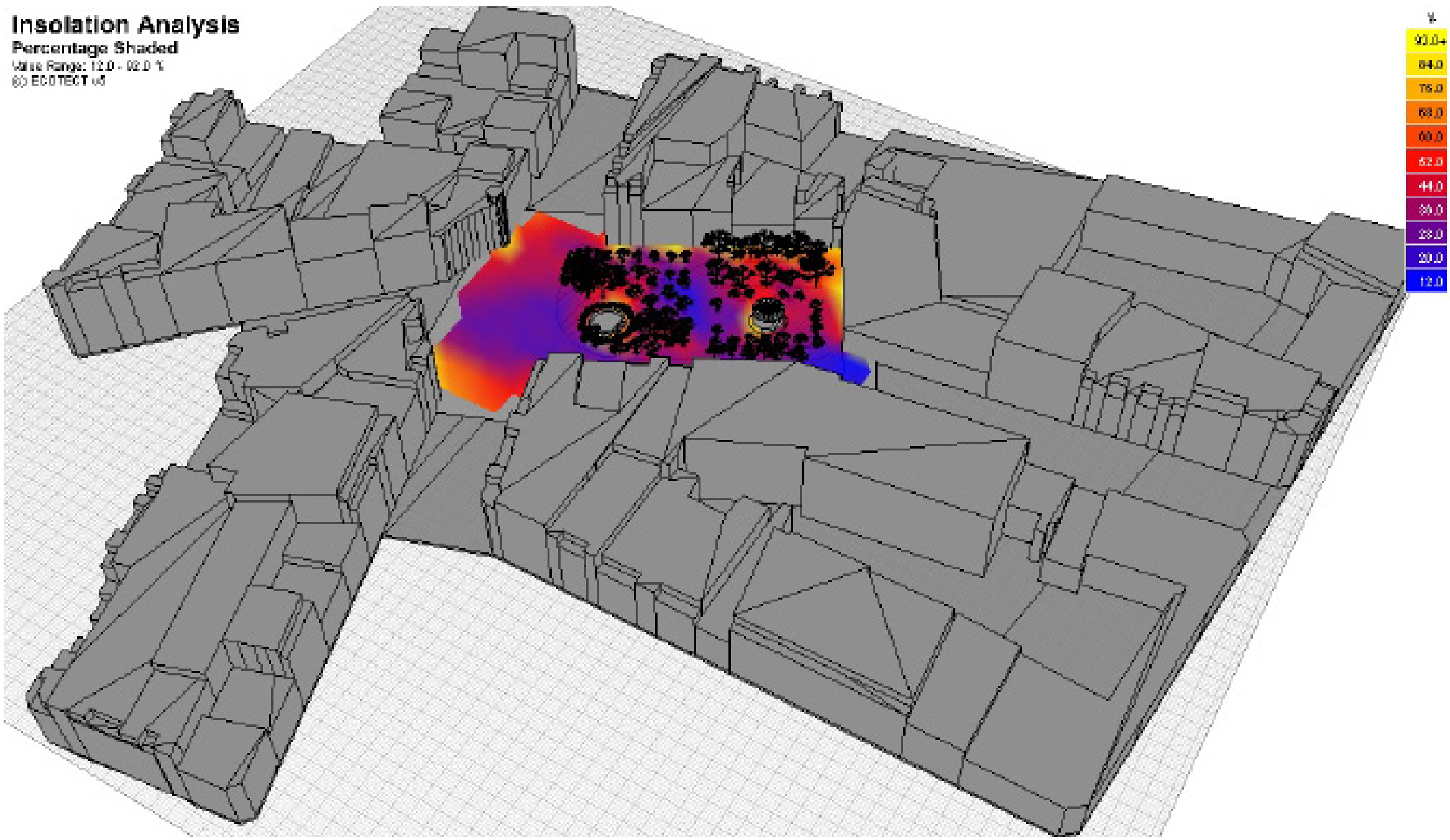

Figura 3. Levantamiento 3D de la zona estudiada y simulación de la disponibilidad de sombra (\%).

Fuente: Elaboración propia
A c. Medición local de condiciones climáticas. En 3 días de los meses de abril, mayo y junio (29 de abril, 27 de mayo y 24 de junio), se realizaron mediciones de las condiciones climáticas en la plaza de Chamberí. En estos meses, las condiciones climáticas exteriores de Madrid pasan de unas temperaturas intermedias a unas condiciones más extremas de calor. Se realizaron mediciones de la temperatura superficial de materiales de pavimentado, fachadas y mobiliario. Se midieron también las condiciones higrotérmicas exteriores y la velocidad del viento. Los datos se tomaron tanto al sol como a la sombra. Los días de toma de mediciones tenían unas condiciones ambientales similares (días despejados). Se realizaron a las 9 a.m., 4:30 p.m. y 10:00 p.m. hora local (UTC+1). Dado que en el espacio público el intercambio de energía entre materiales y habitantes se da a escala de microclima, las mediciones se realizaron a una altura del suelo de entre 1.5 y $2 \mathrm{~m}$.

Se empleó un equipo Testo 400 con 3 sensores: Sonda de temperatura seca del aire $\left({ }^{\circ} \mathrm{C}\right)$ y humedad relativa $(\%)$, sensor de temperaturas de contacto $\left({ }^{\circ} \mathrm{C}\right)$, sonda termoanemométrica de velocidad de viento $(\mathrm{m} / \mathrm{s})$ y temperatura del aire $\left({ }^{\circ} \mathrm{C}\right)$. En paralelo se realizaron termografías con cámara térmica FLIR para la medición de la radiación infrarroja de onda larga y comprobación de la buena elección de los puntos de medida.

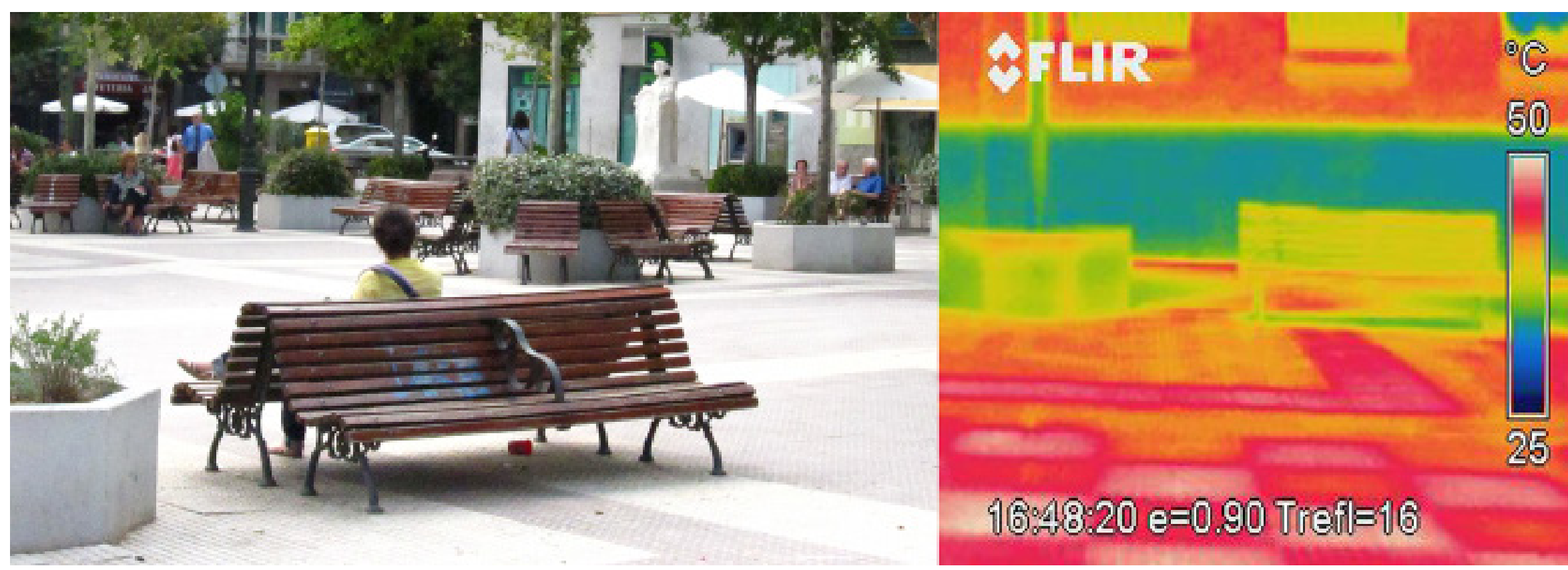


e. El registro de personas y actividades se dio simultáneamente, mediante observación no intrusiva, para la identificación del número de personas, la actividad estancial, edad y localización. La localización de personas y registro de actividades se realizó simultáneamente a las mediciones higrotérmicas para poder establecer posibles relaciones entre las condiciones ambientales y el uso que hacen los ciudadanos del espacio. Los criterios empleados fueron los siguientes:

1. Tan sólo se han identificado las personas que realizan una actividad estancial, sin registrar las personas que tan sólo pasan por estos espacios o permanecen en la misma menos de 10 minutos.

2. Se identifican sólo las personas localizadas en zonas públicas, sin contabilizar a los ciudadanos que hacen uso de las terrazas de bares y restaurantes, ya que su utilización del espacio urbano está más condicionada por los equipamientos que por los condicionantes climáticos o de diseño.

3. Se distinguen las diversas actividades metabólicas: personas sentadas, de pie 0 jugando/realizando actividades dinámicas.

4. Se identifica la edad de las personas.

5. Se han diferenciado las personas al sol y a la sombra, considerando sombra tanto la que es arrojada por edificios o elementos constructivos, como la sombra de los árboles.

Todos estos criterios se han representado gráficamente del siguiente modo:

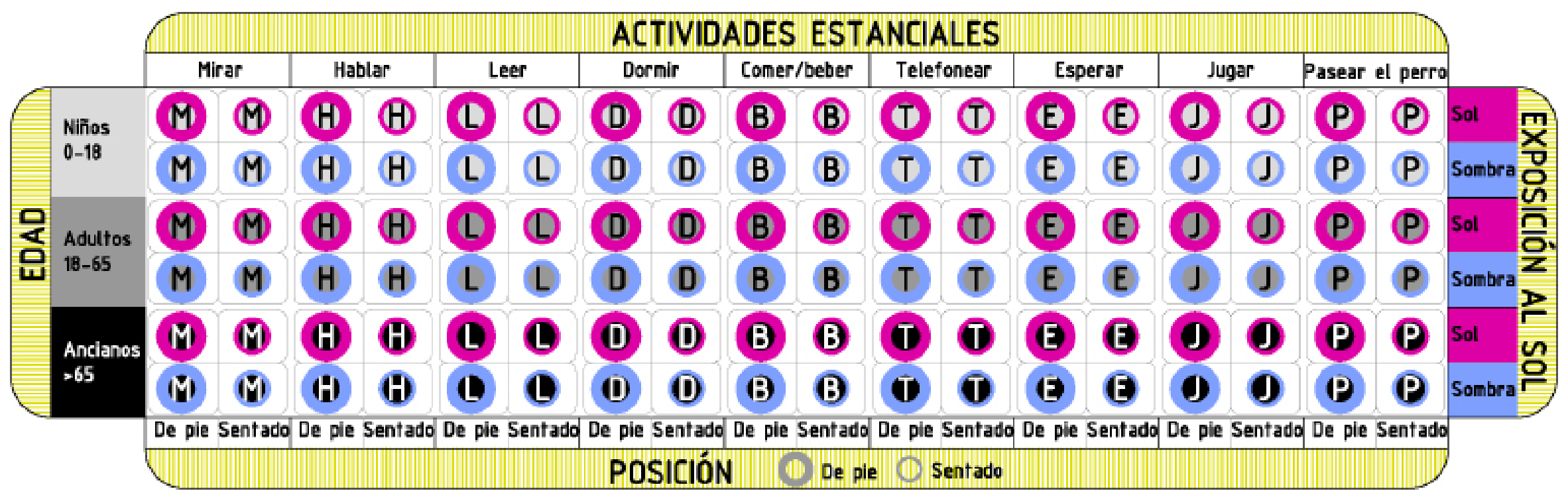

Figura 5. Leyenda del registro de personas: localización, actividad, edad y posición. Fuente: Elaboración propia.
Procesado del estudio bioclimático, las mediciones ambientales y simulaciones

a. Estudio climático: Con base al estudio de datos climáticos de las bases estatales, se definen de estrategias necesarias para alcanzar el confort higrotérmico a lo largo de los meses estudiados.

b. Mediciones y simulaciones: Por una parte, se procesan las mediciones de las condiciones microclimáticas, comparando temperaturas y humedades relativas en diversas situaciones de soleamiento en zonas vegetadas y junto a fuentes de agua. Por otra, se establece el comportamiento térmico de los materiales con base a sus propiedades, su localización en el espacio y con diversas condiciones de soleamiento. Mediante las simulaciones se establecen las superficies con mayor y menor captación de energía solar, así como los espacios permanentemente soleados y sobreados.

c. Localización de las personas: El análisis de los datos recabados permite:

1. Conocer el uso y la localización en el espacio con base a indicadores como la edad y el sexo.

2. Se establecen las actividades más realizadas y su relación con la edad.

3. Las posturas corporales adoptadas en el espacio y su relación con la edad.

4. El tipo de relaciones.

5. La época y la hora del día con mayor y menor afluencia de gente a la plaza. 
Figura 6. Vista parcial de la plaza Chamberí. Fuente: Elaboración propia.
$1 \quad$ Granito gris claro (reflectancia $(\mathrm{R})$ : $0,60)$, densidad ( $p$ ): $2500-2700 \mathrm{~kg} / \mathrm{m}^{3}$, factor de resistencia al paso de vapor de agua (n) 10.000 calor especifico (cp) 1000Jkg-1K-1, emisividad $(\varepsilon)$ $\left(20^{\circ} \mathrm{C}\right) 0,45$, absortividad (a) 0,45, conductividad térmica $(\lambda) 3,5 \mathrm{~W} / \mathrm{mK}$

\section{P: $1300 \mathrm{~kg} / \mathrm{m}^{3}, \mathrm{R}: 0,85, \mathrm{n:} 10, \mathrm{cp}: 1000$} J•kg-1 ${ }^{-1}{ }^{-1}$ y $\varepsilon\left(24^{\circ} \mathrm{C}\right): 0,92, a: 0,26, \lambda: 0,53 \mathrm{~W} / \mathrm{mK}$

3 P: $2300 \mathrm{~kg} / \mathrm{m}^{3}, \mathrm{R}: 0,35, \mathrm{n}: 10, \mathrm{cp}:$ 1000Jkg-1K-1, $\varepsilon\left(21^{\circ} \mathrm{C}\right): 0,93, a: 0,7, \lambda: 0,814 \mathrm{~W} /$ $\mathrm{mK}$

$4 \quad$ D: $2600 \mathrm{~kg} / \mathrm{m} 3, \mathrm{cp}: 1000 \mathrm{Jgg}^{-1} \cdot \mathrm{K}^{-1}, \lambda: 3,5$ W/mK, n: 10.000

$5 \quad$ Pino densidad media $\left(500 \mathrm{~kg} / \mathrm{m}^{3}\right)$, R: 0,29 n: 20, cp: $1600 \mathrm{Jkg}^{-1} \mathrm{~K}^{-1}, \varepsilon\left(70^{\circ} \mathrm{C}\right): 0,94, \mathrm{a}: 0,92, \mathrm{\lambda}$ $0,148 \mathrm{~W} / \mathrm{mK}$
Conclusiones respecto al carácter climático del espacio y comportamiento de las personas.

a. Datos climáticos teóricos y mediciones locales: Relación y divergencia entre mediciones in-situ en la estación meteorológica en la plaza de Chamberí y la estación meteorológica estatal en Parque del Retiro, cuyos datos se emplean como bases para estudios bioclimáticos y bases climáticas internacionales. Se determina numéricamente el efecto de Isla de Calor Urbana en la plaza.

b. Condiciones microclimáticas y comportamiento térmico de los materiales.

c. Mediciones ambientales y uso del espacio: Se establecen relaciones entre las condiciones microclimáticas, la temperatura de los materiales y el uso del espacio. Además, se determinan los espacios que no han sido empleados en ningún momento a lo largo del estudio realizado y aquellos que triunfan más en relación con las características físicas y térmicas de la plaza.

\section{Caso de estudio}

La plaza de Chamberí es un espacio tradicional localizado al norte de las últimas murallas de Madrid, en el ensanche del siglo XIX. La plaza dispone de equipamientos educacionales, religiosos, sociales, hosteleros y comercios, permitiendo a los vecinos realizar actividades, funciones y compras necesarias del día a día sin necesidad de trasladarse. Estos usos están directamente situados en la planta baja de los edificios, por lo que son fácilmente accesibles. En la plaza hay zonas estanciales, una zona rectangular de arena con zona de juego de niños, un quiosco de música en el centro, una fuente en la esquina sur, aparcamientos viales rodados al sur y al oeste, y arbolado y pequeños jardines. La plaza tiene bancos suficientes, distribuidos por toda la plaza (Whyte, 1980).

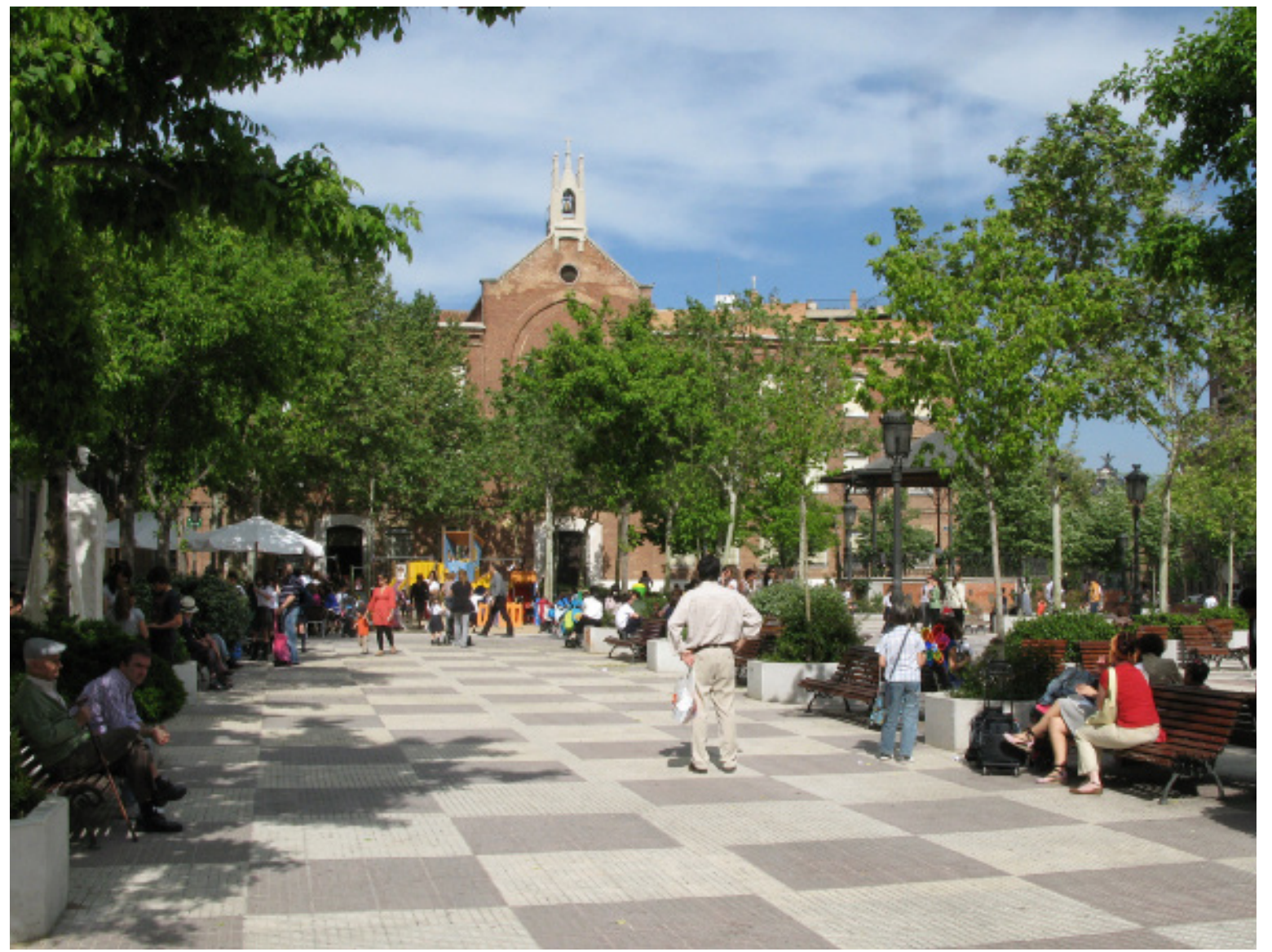

Tiene unas dimensiones de $48 \times 87 \mathrm{~m} 2$ y está localizada en un hueco urbano de $71 \times 121 \mathrm{~m} 2$.

Los materiales de acabado son granito gris claro ${ }^{1}$, encalados blancos ${ }^{2}$ y ladrillo macizo rojo ${ }^{3}$ en fachadas, suelos de terrazos rojizos y blancos grisáceos ${ }^{4}$ y arena. Los bancos son de madera ${ }^{5}$. Los datos de los materiales se extraen del Código Técnico de la Edificación (Ministerio de Fomento, 2010). 


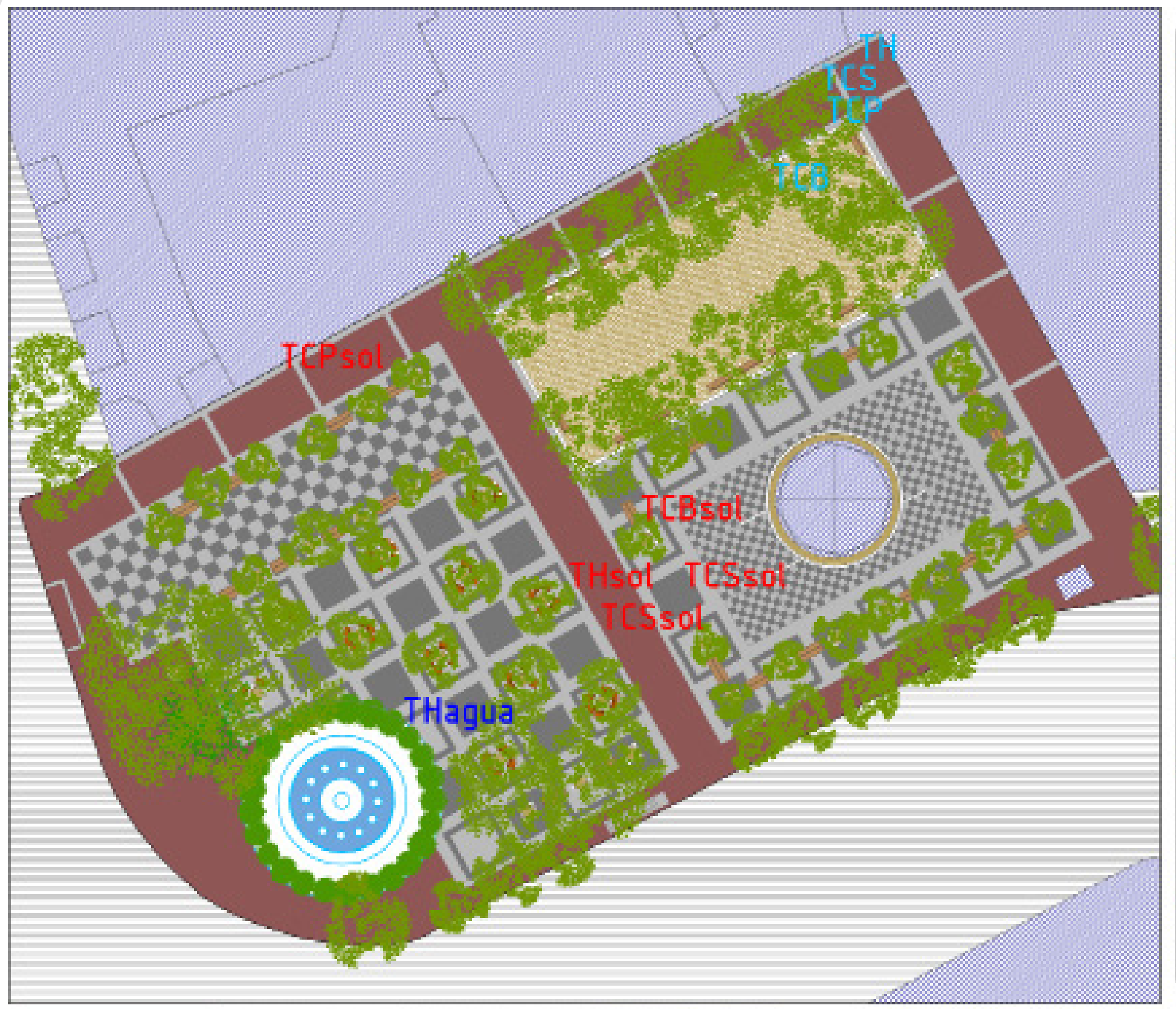

GEFEMDA

THsol

Temperatura y

hiunedad al sol

TH:

Temperatura y

humedad a la

sombra

THagia:

Temperatura y

humedad junta a

agua

TCS601:

Tempratura de

contacto del suelo

al sol

TCPsol:

Temperatura de

contacto de

fachadas all sol

TGBsol:

Temperatura de

contacta do hanco

al 50

TCS:

Tempratura de

contacto del suelo

a la sumbera

ILP:

Temperatura de

conntactó de

fachadas a la

sombra

TiCB:

Temperatura de

contacto de banco

a la sunbra

\begin{tabular}{|c|c|c|c|c|c|c|}
\hline & & & & & & \\
\hline TERADO ROJLO & TEAHADO BLANLO & TEAHARO GHIS & MELA & CALZADA RUDADA & CONSTGUCODES & FUENTE \\
\hline 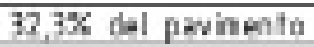 & 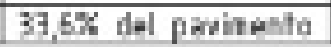 & 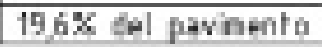 & 16,55 del pimitimita & OK its pinitimeta & G neñants & 1 \\
\hline
\end{tabular}
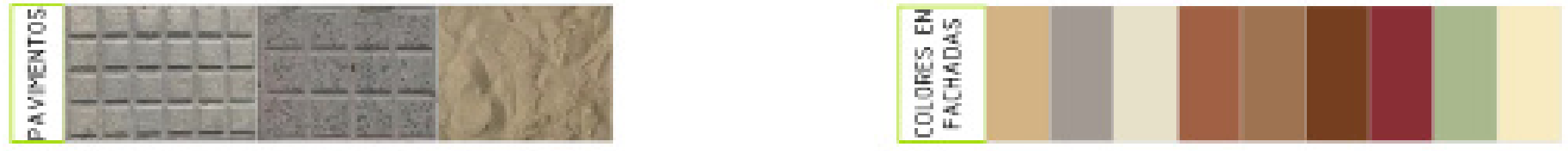

Figura 7. Caracterización física y localización de las mediciones. Fuente: Elaboración propia.

\section{Resultados}

\section{Estudio climático}

Durante el mes de abril, con un arropamiento de 1clo e independientemente de la actividad estancial que se esté realizando (caminar, estar de pie o estar sentado), será necesaria una aportación de calor para alcanzar el bienestar higrotérmico.

En mayo seguirá siendo necesaria la aportación solar para alcanzar el bienestar hasta las 4:00 p.m. si se está sentado, hasta las 3:00p.m. si se está de pie o hasta la 1:00p.m. si se camina. Se alcanzarán ciertas horas de bienestar a primeras horas de la tarde (entre 3 y 4 horas de confort (Neila, 2004)) si se está sentado o de pie y 2 horas a primera y última hora de la tarde si se camina. Comenzará a resultar necesario disponer de espacios sombreados por los que se pueda caminar o jugar para la mayor parte de las horas de la tarde.

Durante el mes de junio, la estrategia de sombreamiento resultará necesaria a partir del mediodía y durante toda la tarde, incluso con una reducción de arropamiento a $0,7 \mathrm{clo}$. Puntualmente, a media tarde será necesario el movimiento de aire para alcanzar el confort caminando o jugando. Tan sólo resulta necesaria una aportación solar ligera hasta mediodía si se está sentado o de pie y hasta las 10:00a.m. si se camina. Entre las 12:00a.m. y las 1:00p.m. si se está sentado o de pie y entre las 10:00a.m. y las 
11:00a.m. si se está caminando, se alcanzará el bienestar ampliado (hasta un 20\% de personas insatisfechas).

En los momentos más cálidos, la humedad relativa (HR) baja del $30 \%$, pero no baja del $20 \%$ en el periodo analizado, por lo que no resulta estrictamente necesaria la aportación de humedad al ambiente.
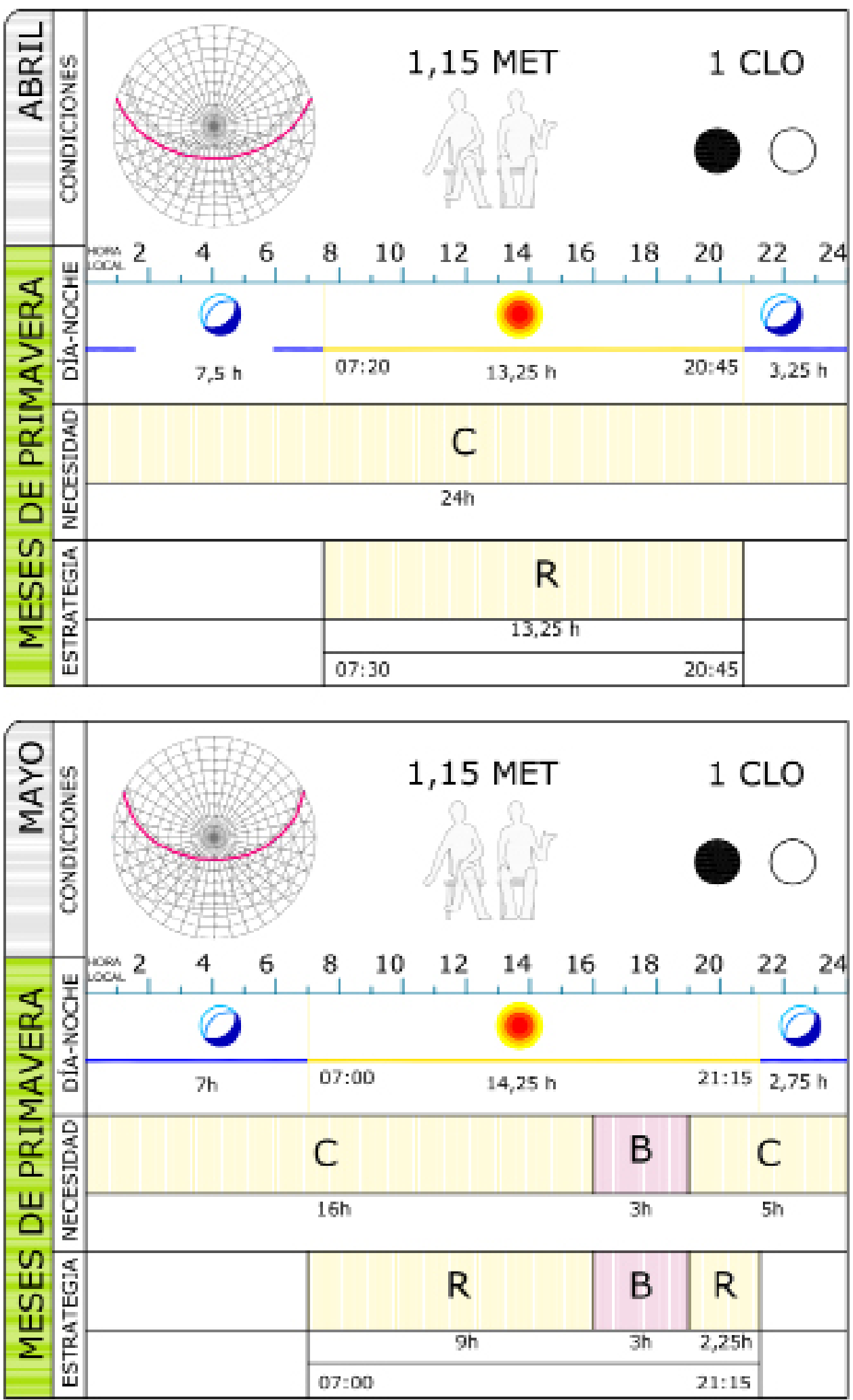

Figura 8. Necesidades y estrategias bioclimáticas: C: aportación de calor/ E: reducción de calor/ B: bienestar ampliado/ $\mathrm{B}+\mathrm{S}$ : bienestar con sombreamiento/ R: espacios con radiación solar directa/ S: espacios sombreados/ $\mathrm{S}+\mathrm{V}$ : espacios sombreados y ventilados. Fuente: Elaboración propia.

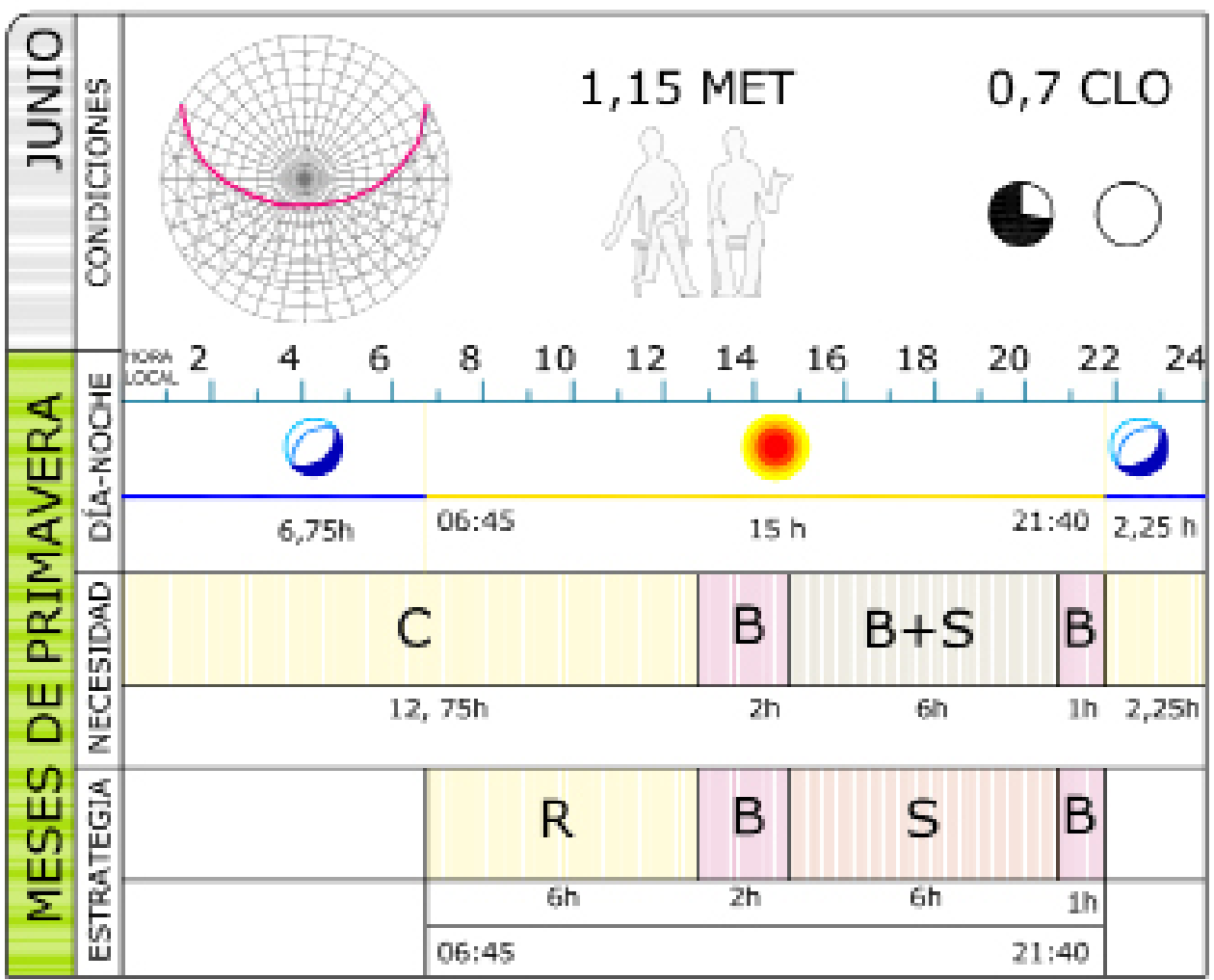



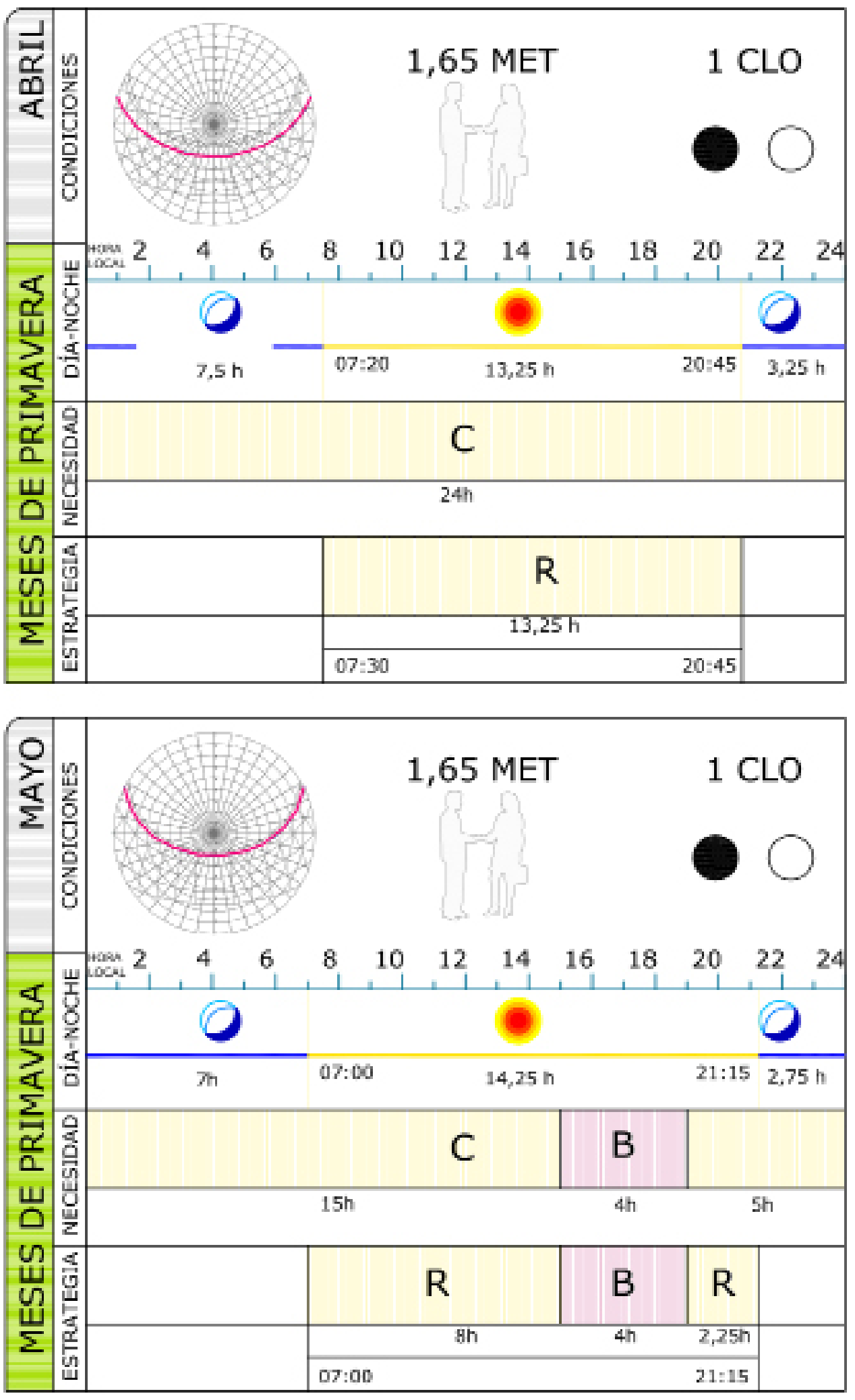

Figura 8. Necesidades y estrategias bioclimáticas: C: aportación de calor/ E: reducción de calor/ B: bienestar ampliado/ B+S: bienestar con sombreamiento/ R: espacios con radiación solar directa/ S: espacios sombreados/ $\mathrm{S}+\mathrm{V}$ : espacios sombreados y ventilados. Fuente: Elaboración propia.

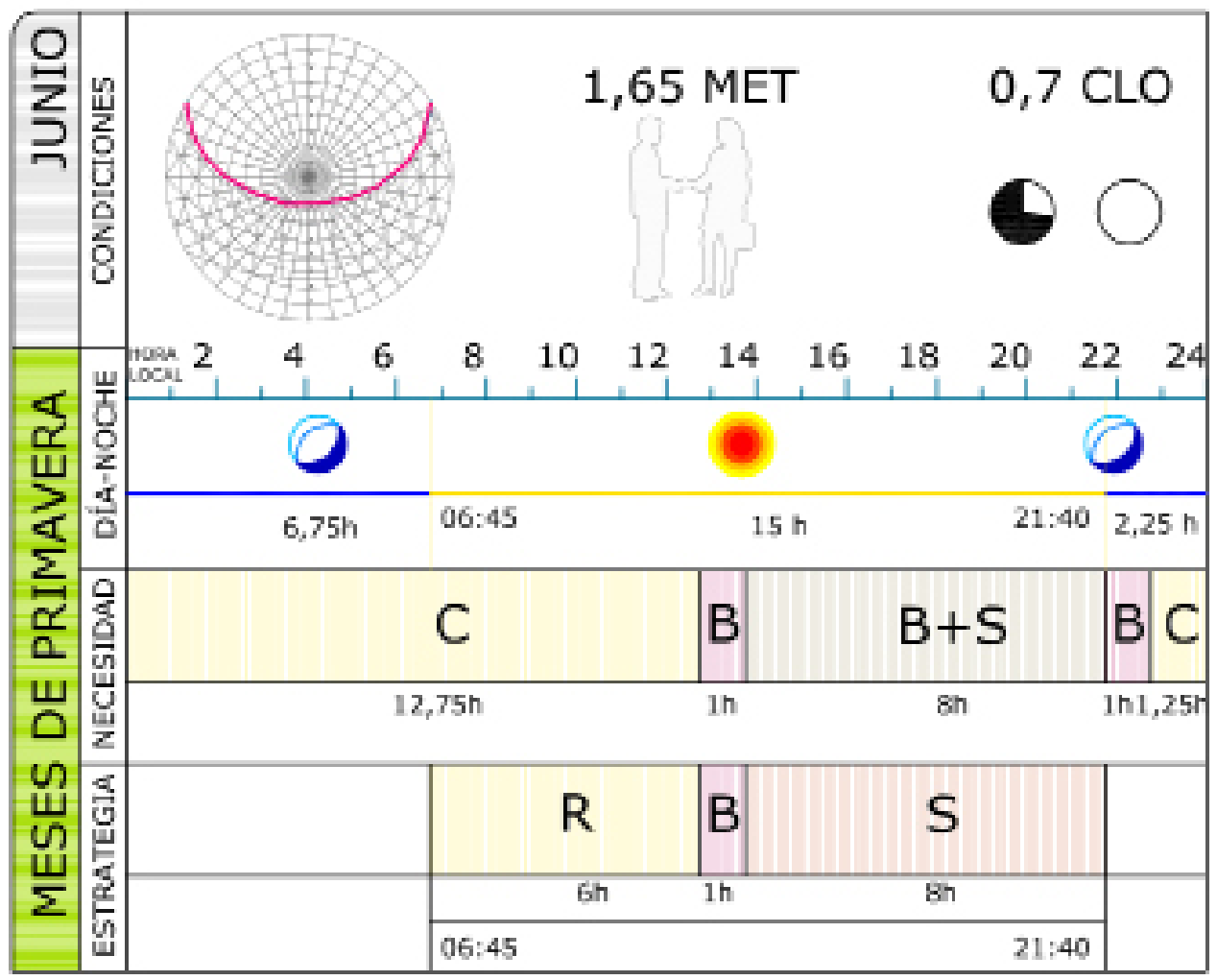



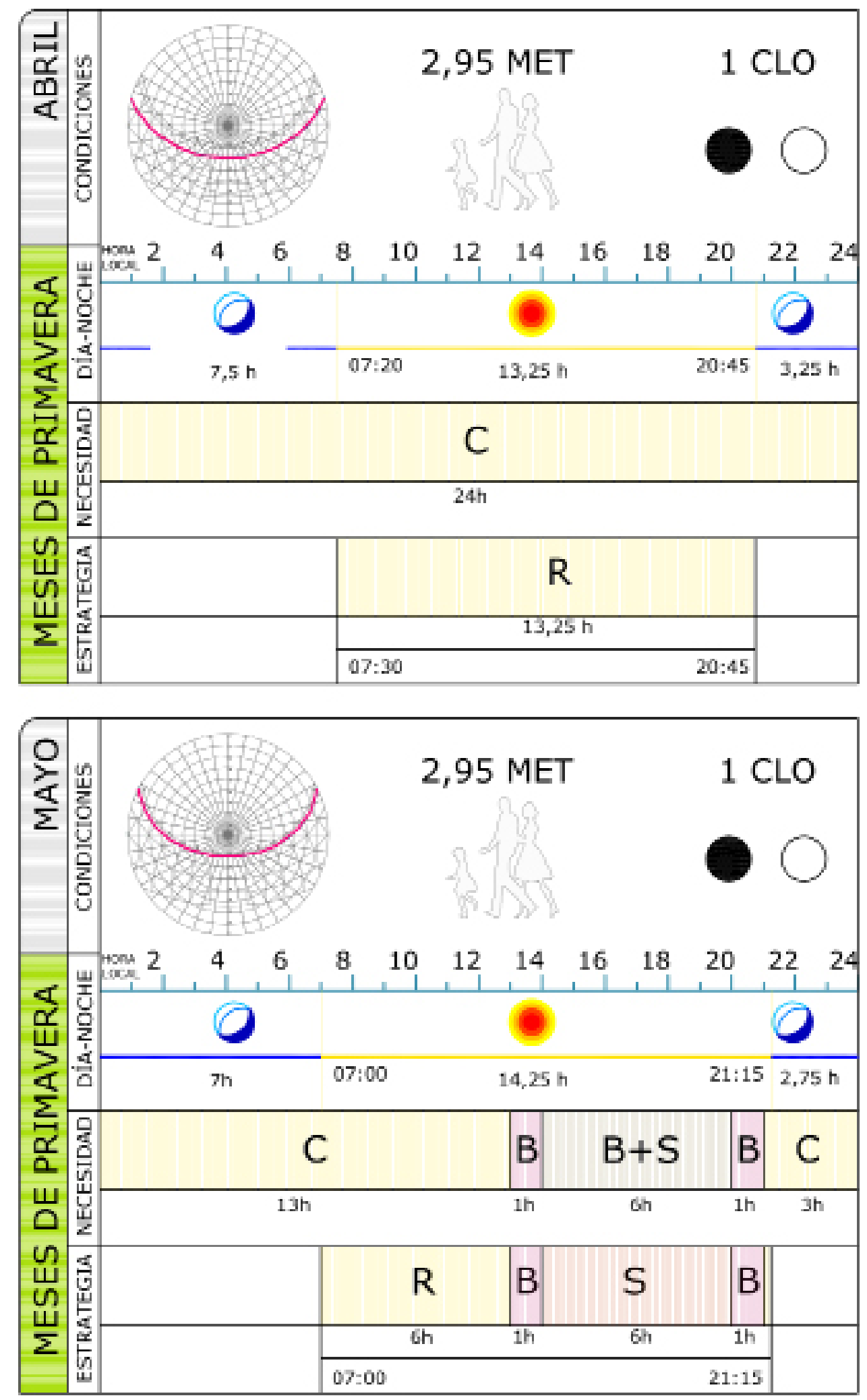

Figura 8. Necesidades y estrategias bioclimáticas: C: aportación de calor/ E: reducción de calor/ B: bienestar ampliado/ B+S: bienestar con sombreamiento/ R: espacios con radiación solar directa/S: espacios sombreados/ $\mathrm{S}+\mathrm{V}$ : espacios sombreados y ventilados. Fuente: Elaboración propia.

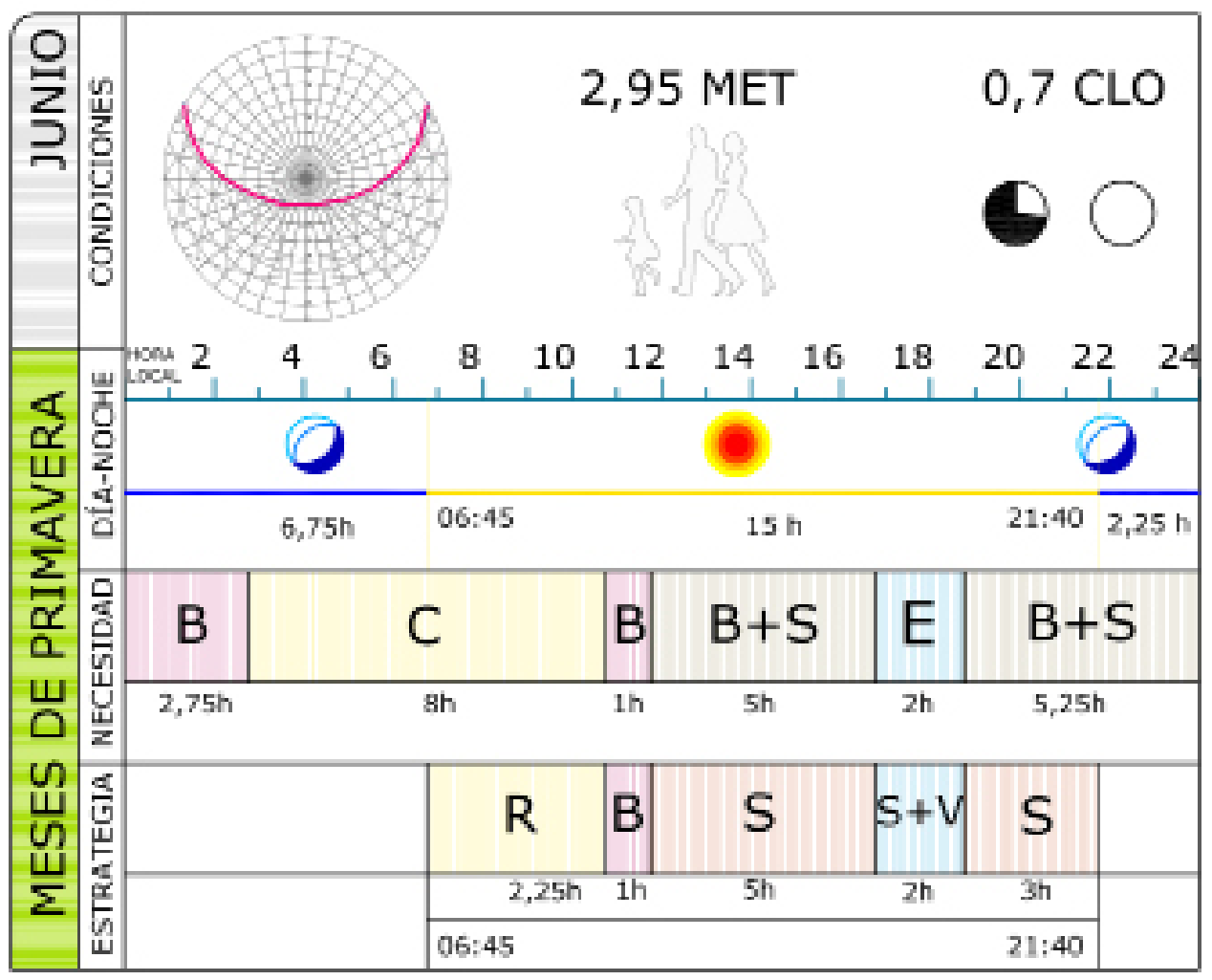




\section{Mediciones in-situ y simulaciones}

Analizando las mediciones de temperatura del aire (Ta), se observa que la evolución de temperatura y humedad relativa es similar en los meses de abril y mayo, siendo éste un poco más seco. Sin embargo, las temperaturas se incrementan notablemente en junio, pasando de máximas de $27-28^{\circ} \mathrm{C}$ a una máxima de $33,4^{\circ} \mathrm{C}$. La humedad relativa (HR) desciende también notablemente, alcanzando mínimas de $24,5 \%$.

Existen diferencias de Ta notables entre los espacios soleados y sombreados en la hora de máximo calor: entre 2 y $2,5^{\circ} \mathrm{C}$ en abril y mayo y $1^{\circ} \mathrm{C}$ menos en junio a la sombra. La HR también es mayor en los espacios a la sombra (entre un 1,5 y $3 \%$ ).

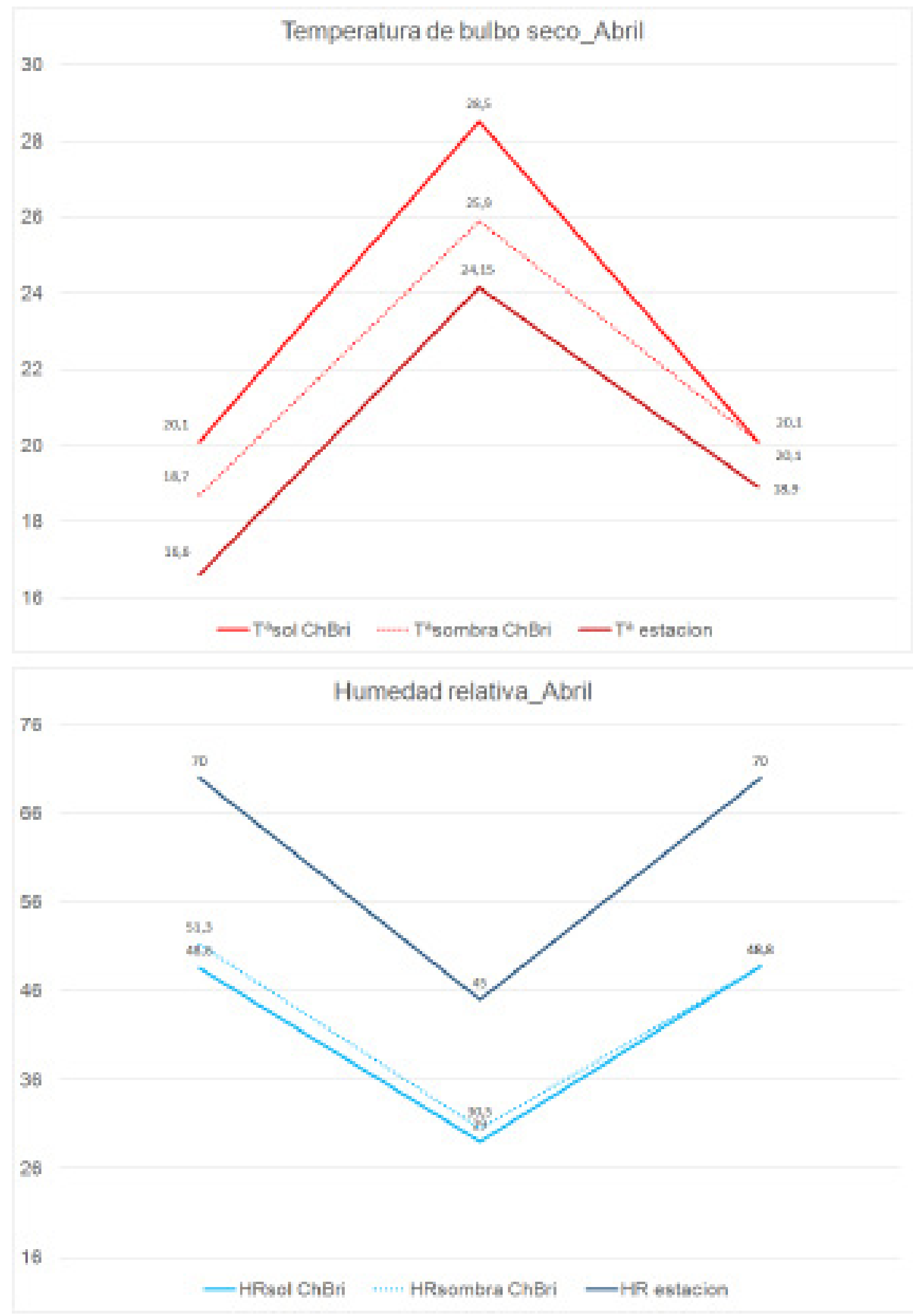


Figura 9. Mediciones de temperaturas del aire de bulbo seco y humedad relativa al sol y a la sombra (9:00a.m., 4:30p.m. y 10:00p.m.) y datos de la estación meteorológica de Retiro.

Fuente: Elaboración propia.

\section{Temperatura de bulbo seco_Mayo}

211

26

24

22

20

11

16 19:4

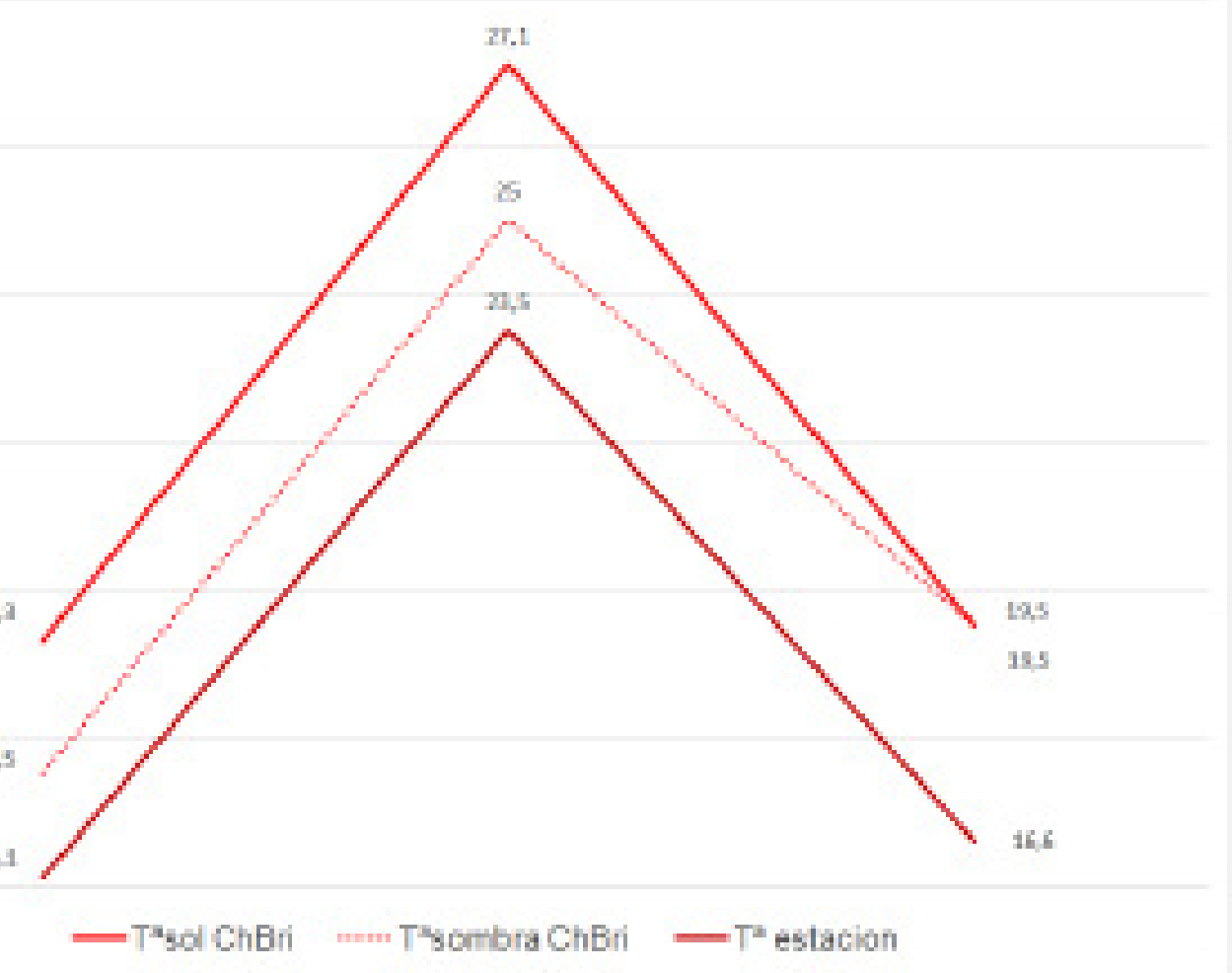

Humedad relativa_Abril

76

69

58

46

36

26

$1 \mathrm{E}$ 
Figura 9. Mediciones de temperaturas del aire de bulbo seco y humedad relativa al sol y a la sombra (9:00a.m., 4:30p.m. y 10:00p.m.) y datos de la estación meteorológica de Retiro.

Fuente: Elaboración propia.

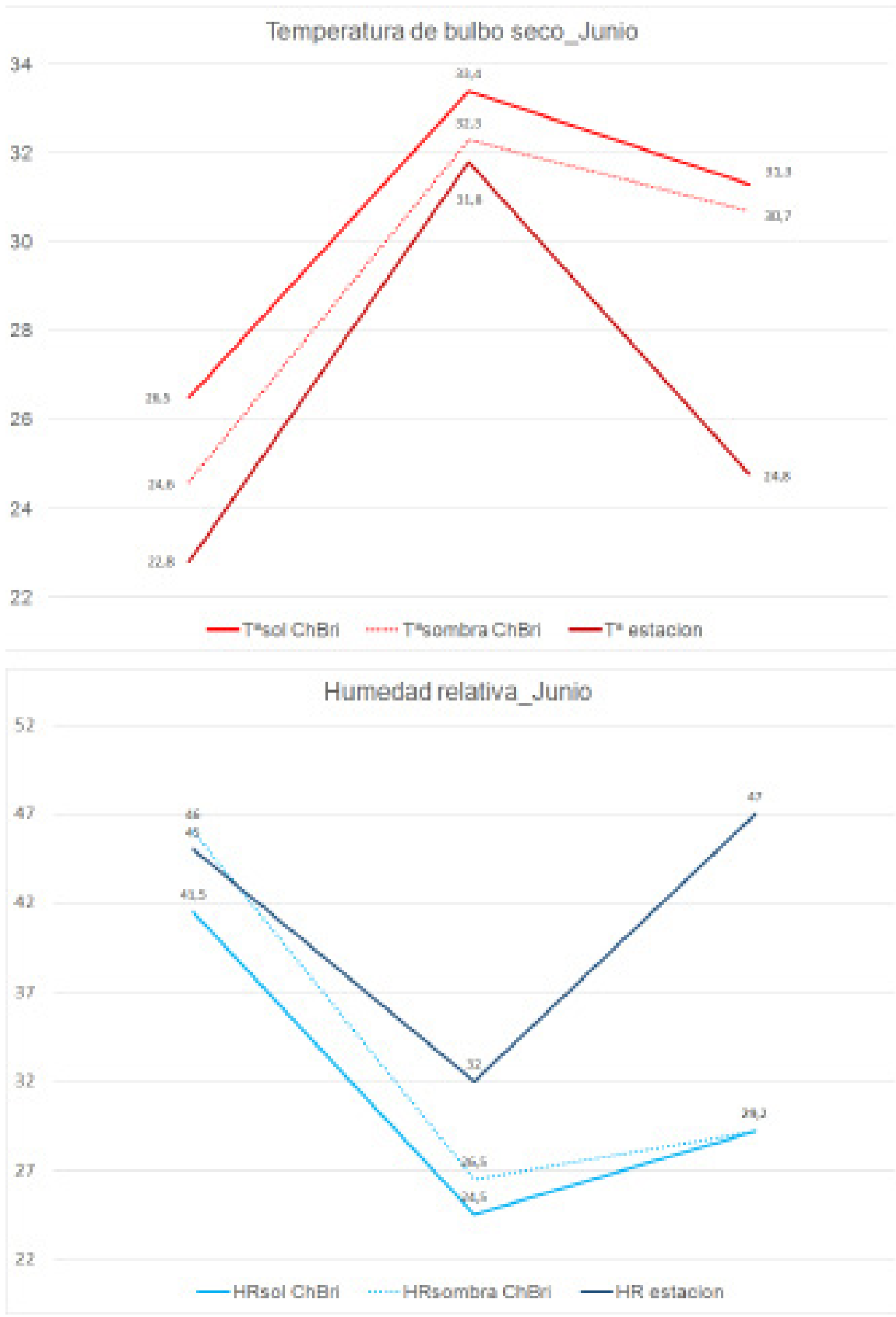

En las mediciones realizadas al sol junto a la fuente de la plaza Chamberí, se registró un incremento de la $\mathrm{HR}$ de entre un $1 \%$ y un $3 \%$. La velocidad del viento media ha sido siempre inferior a los $2 \mathrm{~m} / \mathrm{s}$.

Las temperaturas superficiales (TC) de los materiales varían con base a sus propiedades y su localización en el espacio. Las temperaturas más elevadas se dan en los suelos de terrazo rojo, alcanzando una TC al sol de $50,6^{\circ} \mathrm{C}$ en la tarde de junio. Los terrazos blancos grisáceos se mantienen con temperaturas inferiores dado su mayor albedo, con una TC máxima de $46,3^{\circ} \mathrm{C}$ en ese mismo momento. Los mismos pavimentos registran TC de hasta $10^{\circ} \mathrm{C}$ inferiores al encontrarse a la sombra. Las fachadas siguen la misma dinámica en las encaladas de blanco, pues se registran hasta un máximo de $8^{\circ} \mathrm{C}$ menos que en las fachadas de ladrillo caravista. 


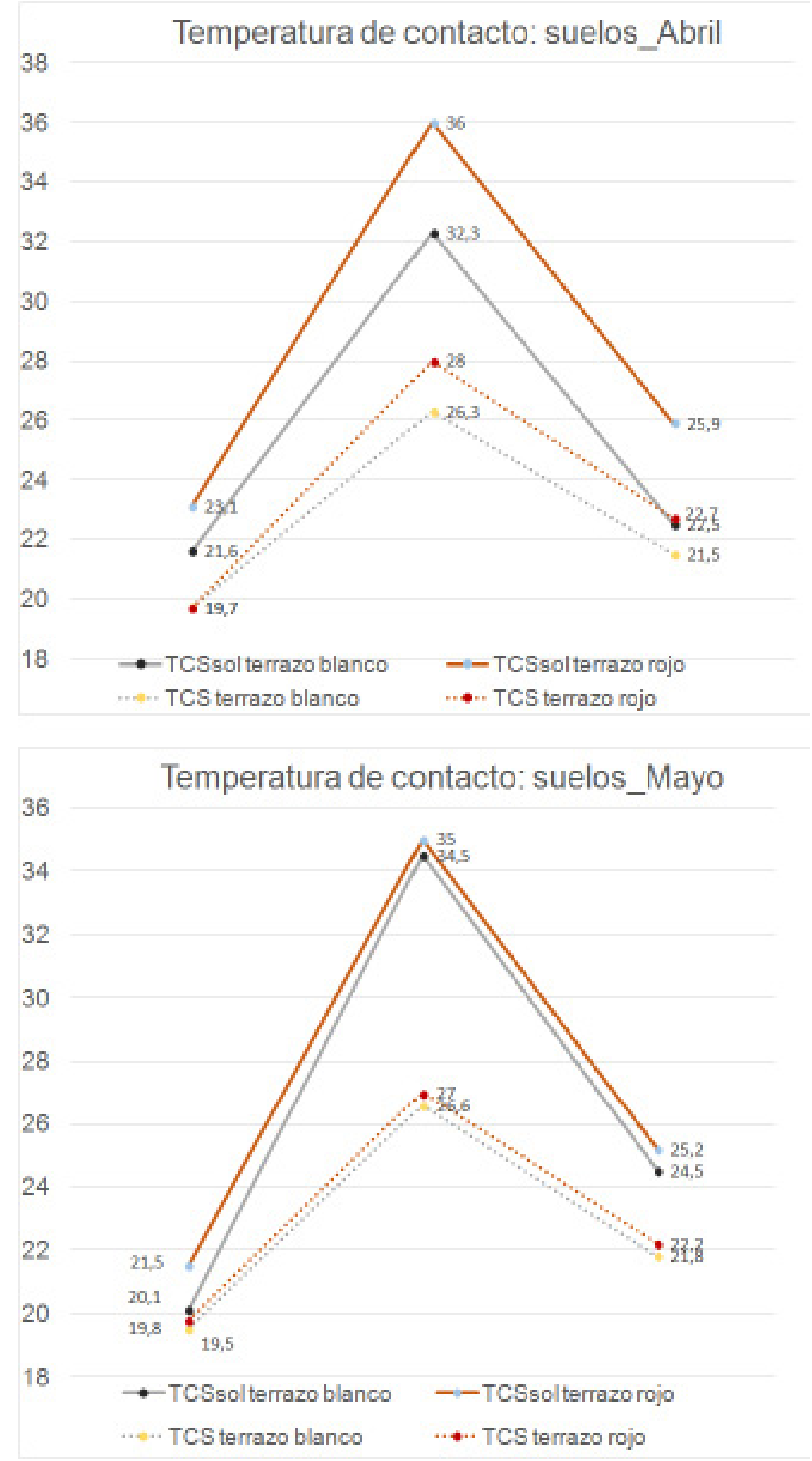

Figura 10. Temperaturas superficiales al sol y a la sombra de los pavimentos (9:00a.m.

Fuente: Elaboración propia. 
A excepción de la madera, durante las mañanas y las noches, el comportamiento térmico de los materiales es similar con diferencias máximas entre materiales entre $2^{\circ}$ $\mathrm{C}$ y $4^{\circ} \mathrm{C}$. Mantienen lógicamente, además, una TC similar a la Ta, tan sólo ligeramente superior sin superar los $3^{\circ} \mathrm{C}$ de diferencia. Sin embargo, en las horas centrales del día, la TC de los materiales será entre $4^{\circ} \mathrm{C}$ y $13^{\circ} \mathrm{C}$ superior a la Ta. Los materiales por la noche aún conservan energía almacenada, manteniendo una TC superior en unos $4^{\circ} \mathrm{C}$ a la TC de la mañana, causa fundamental de la ICU. El efecto de isla térmica está estrechamente relacionado con los materiales de construcción (Hui, 2015), pues los materiales empleados tienen gran capacidad de almacenamiento de calor. Los materiales de construcción absorben, almacenan, reflejan y emiten energía radiante, intercambiando así calor con el aire e influyendo en la temperatura del aire cercano a la superficie (near-surface temperatures). Es en la Urban Canopy Layer (UCL) donde este calentamiento de la atmósfera urbana se hace más patente (Oke, 1982), condicionando así el confort térmico exterior de los ocupantes del espacio público (Gaitani, Mihalakakou \& Santamouris, 2007), así como de las actividades económicas, sociales y de ocio que allí se realizan.

\section{Localización de personas y actividades}

Uno de los aspectos más complejos de caracterizar es el perfil del usuario. El intentar generalizar esa información es una tarea infructuosa que genera graves errores, por lo que es necesario realizar un estudio específico. En las plazas seleccionadas prevalece el carácter doméstico, esto es, un uso cotidiano de este espacio por parte de los vecinos. Se han contabilizado un total de 686 personas con $265(38,6 \%)$ niños, 340 $(49,6 \%)$ adultos y $81(11,8 \%)$ adultos mayores. Las actividades que se han desarrollado en la plaza son: mirar lo que sucede alrededor, hablar con otras personas, leer, dormir, comer, beber, hablar o consultar el teléfono, esperar a alguien, jugar y pasear al perro.

La actividad que más triunfa en esta plaza es la de hablar con alguien. Tras esta actividad, la que más se realiza es la de mirar lo que sucede alrededor o lo que hacen los demás. La tercera actividad más realizada en la plaza es la de jugar. La actividad principal de los niños está relacionada con el juego, aunque también se les encuentra hablando con personas de diferentes edades, durmiendo, comiendo y bebiendo. Las personas entre 18 y 65 años son aquellas que realizan actividades más diversas, aunque la más habitual es la de hablar con alguien. Las personas mayores cuando están solas realizan dos actividades principales: hablar cuando se encuentran acompañadas, o mirar lo que sucede a su alrededor o lo que hacen los demás. Las actividades que se desarrollan se realizan principalmente en posición sentada, aunque hay una gran diferencia entre los niños que permanecen y realizan sus actividades mayoritariamente en pie y los adultos y ancianos que lo hacen sentados.

La plaza se emplea por ciudadanos de todas las edades y sexos: por la mañana se encuentran principalmente personas adultas (entre el $70 \%$ y el $85 \%$ de las personas), pero por la tarde se pueden encontrar muchos niños, llegando en ocasiones a superar al número de adultos. Las relaciones que se dan en la plaza son tanto personales (familias, amigos o cuidadoras con niños) como no personales (público en general 0 grupos específicos como los escolares). 


\begin{tabular}{|c|c|c|c|c|c|c|c|}
\hline \multicolumn{2}{|c|}{ NÚMERO DE USUARIOS } & \multicolumn{6}{|l|}{29 ABRIL } \\
\hline & NÚMERO DE PERSONAS & TOTAL & 0-18 AÑOS & 18-65 AÑOS & $>65$ AÑOS & $\begin{array}{l}\text { A C T I V I D A D E S } \\
\text { PRINCIPALES }\end{array}$ & ARROPAMIENTO \\
\hline \multirow{5}{*}{ 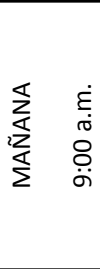 } & TOTAL & $7(100 \%)$ & $0(0 \%)$ & $6(85,7 \%)$ & $1(14,3 \%)$ & \multirow{5}{*}{$\begin{array}{l}\text { Hablar (4p) } \\
\text { Mirar (3p) }\end{array}$} & \multirow[t]{13}{*}{1 CLO } \\
\hline & AL SOL & $6(85,7 \%)$ & 0 & 5 & 1 & & \\
\hline & SOMBRA & $1(14,3 \%)$ & 0 & 1 & 0 & & \\
\hline & EN PIE & $0(0 \%)$ & 0 & 0 & 0 & & \\
\hline & SENTADO & $7(100 \%)$ & 0 & 6 & 1 & & \\
\hline \multirow{5}{*}{ 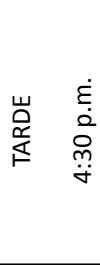 } & TOTAL & $271(100 \%)$ & $136(50 \%)$ & $118(43,7 \%)$ & $17(6,3 \%)$ & \multirow{5}{*}{$\begin{array}{l}\text { Hablar (130p) } \\
\text { Jugar (96p) } \\
\text { Mirar (29p) }\end{array}$} & \\
\hline & AL SOL & $117(43,2 \%)$ & 70 & 43 & 4 & & \\
\hline & SOMBRA & $154(56,8 \%)$ & 66 & 75 & 13 & & \\
\hline & EN PIE & $138(50,9 \%)$ & 103 & 35 & 0 & & \\
\hline & SENTADO & $133(49,1 \%)$ & 33 & 83 & 17 & & \\
\hline \multirow{3}{*}{ 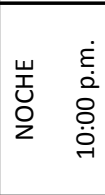 } & TOTAL & $45(100 \%)$ & $31(68,9 \%)$ & $11(24,4 \%)$ & $3(6,7 \%)$ & \multirow{3}{*}{$\begin{array}{l}\text { Hablar (22p) } \\
\text { Jugar (14p) } \\
\text { Mirar (9p) } \\
\end{array}$} & \\
\hline & EN PIE & $25(55,6 \%)$ & 21 & 4 & 0 & & \\
\hline & SENTADO & $20(44,4 \%)$ & 10 & 7 & 3 & & \\
\hline \multicolumn{2}{|c|}{ NÚMERO DE USUARIOS } & \multicolumn{6}{|l|}{27 MAYO } \\
\hline & NÚMERO DE PERSONAS & TOTAL & 0-18 AÑOS & 18-65 AÑOS & $>65$ AÑOS & \begin{tabular}{|l|} 
A C T I V I D A D E S \\
PRINCIPALES
\end{tabular} & ARROPAMIENTO \\
\hline \multirow{5}{*}{ 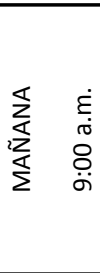 } & TOTAL & $13(100 \%)$ & $1(7,7 \%)$ & $9(69,2 \%)$ & $3(23,1 \%)$ & \multirow{5}{*}{$\begin{array}{l}\text { Hablar (4p) } \\
\text { Mirar (3p) } \\
\text { Jugar (2p) }\end{array}$} & \multirow[t]{13}{*}{$1 \mathrm{CLO}$} \\
\hline & AL SOL & $10(76,4 \%)$ & 0 & 7 & 3 & & \\
\hline & SOMBRA & $3(23,1 \%)$ & 1 & 2 & 0 & & \\
\hline & EN PIE & $5(38,5 \%)$ & 0 & 4 & 1 & & \\
\hline & SENTADO & $8(61,5 \%)$ & 1 & 5 & 2 & & \\
\hline \multirow{5}{*}{ 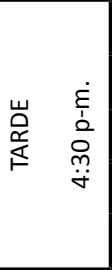 } & TOTAL & $162(100 \%)$ & $62(38,3 \%)$ & $80(49,4 \%)$ & $20(12,3 \%)$ & \multirow{5}{*}{$\begin{array}{l}\text { Hablar (59p) } \\
\text { Mirar (49p) } \\
\text { Jugar (41p) }\end{array}$} & \\
\hline & AL SOL & $63(38,9 \%)$ & 23 & 30 & 10 & & \\
\hline & SOMBRA & $99(61.1 \%)$ & 39 & 50 & 10 & & \\
\hline & EN PIE & $59(36,4 \%)$ & 38 & 18 & 3 & & \\
\hline & SENTADO & $103(63,6 \%)$ & 24 & 62 & 17 & & \\
\hline \multirow{3}{*}{ 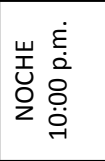 } & TOTAL & $22(100 \%)$ & $2(9,1 \%)$ & $17(49,4 \%)$ & $3(13,6 \%)$ & \multirow{3}{*}{$\begin{array}{l}\text { Hablar (13p) } \\
\text { Mirar (5p) }\end{array}$} & \\
\hline & EN PIE & $1(4,5 \%)$ & 0 & 1 & 0 & & \\
\hline & SENTADO & $21(95,5 \%)$ & 2 & 16 & 3 & & \\
\hline \multicolumn{2}{|c|}{\begin{tabular}{|l|} 
NÚMERO DE USUARIOS \\
\end{tabular}} & \multicolumn{4}{|l|}{24 JUNIO } & & \\
\hline & NÚMERO DE PERSONAS & TOTAL & 0-18 AÑOS & 18-65 AÑOS & $>65$ AÑOS & \begin{tabular}{|l|} 
A C T I V I D A D E S \\
PRINCIPALES
\end{tabular} & ARROPAMIENTO \\
\hline \multirow{5}{*}{ 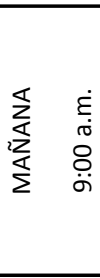 } & TOTAL & $38(100 \%)$ & $1(2,6 \%)$ & $32(84,2 \%)$ & $5(13,2 \%)$ & \multirow{5}{*}{$\begin{array}{l}\text { Hablar (15p) } \\
\text { Mirar (8p) } \\
\text { Jugar (2p) }\end{array}$} & \multirow[t]{13}{*}{0.7 CLO } \\
\hline & AL SOL & $12(31,6 \%)$ & 1 & 8 & 3 & & \\
\hline & SOMBRA & $26(68,4 \%)$ & 0 & 24 & 2 & & \\
\hline & EN PIE & $9(23,7 \%)$ & 1 & 8 & 0 & & \\
\hline & \begin{tabular}{|l} 
SENTADO \\
\end{tabular} & $29(76,3 \%)$ & 0 & 24 & 5 & & \\
\hline & TOTAL & $45(100 \%)$ & $15(33,3 \%)$ & \begin{tabular}{|l|}
$26(57.8 \%)$ \\
\end{tabular} & $4(8,9 \%)$ & Hablar (15p) & \\
\hline & \begin{tabular}{|l|} 
AL SOL \\
\end{tabular} & $10(22,2 \%)$ & 6 & 4 & 0 & Mirar (12p) & \\
\hline 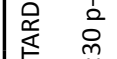 & SOMBRA & $35(77,8 \%)$ & 9 & 22 & 4 & & \\
\hline & \begin{tabular}{|l|} 
EN PIE \\
\end{tabular} & $10(22.2 \&)$ & 8 & 2 & 0 & Jugar (10p) & \\
\hline & SENTADO & $35(77,8 \%)$ & 7 & 24 & 4 & & \\
\hline 8 & TOTAL & $83(100 \%)$ & $17(20,5 \%)$ & $41(49,4 \%)$ & $25(30,1 \%)$ & Hablar (35p) & \\
\hline 总 & EN PIE & $25(30,1 \%)$ & 13 & 9 & 3 & Mirar (24p) & \\
\hline $\begin{array}{l}I_{0}^{2} \\
\mathrm{O}^{2} \\
2\end{array}$ & SENTADO & $58(69,9 \%)$ & 4 & 32 & 22 & Jugar (15p) & \\
\hline
\end{tabular}

Tabla 1. Uso de la plaza. Registro de actividades, número de personas, edad, localización y postura.

Fuente: Elaboración propia.
La plaza se empleó principalmente por la tarde, con muy poca gente por la mañana y algo más por la noche. Esta diferencia en el uso se hace más notable en abril, con 272 personas por la tarde frente a las 7 personas que había por la mañana o las 45 de la noche. En mayo, la diferencia de uso de la plaza sigue siendo evidente con 165 personas contabilizadas por la tarde frente a las 13 de la mañana y las 22 de la noche. En junio, la plaza fue más empleada por la noche, contabilizando tan sólo 45 personas por la tarde, mientras que por la noche la cifra incrementó a 83. 


\section{Discusión}

\section{Divergencia entre clima teórico y mediciones locales registradas}

En los estudios bioclimáticos, se trabaja con datos climáticos resultantes de las medias de entre 10 y 30 años de registro de datos. A nivel internacional, se emplean los archivos .epw de Energy Plus, y a nivel nacional, en España, se dispone de las bases de datos climáticos de la AEMET y las del Código Técnico de la Edificación.

Según el mapa actualizado de la ICU de Madrid (Núñez Peiró, Sánchez-Guevara \& Neila, 2017), existen entre $2^{\circ} \mathrm{C}$ y $3^{\circ} \mathrm{C}$ de diferencia entre la localización de la estación meteorológica más cercana en el Parque del Retiro ${ }^{6}$ y la plaza analizada. Los datos tomados in-situ confirman la ICU al registrarse temperaturas del aire superiores en el mismo periodo en las plazas con respecto a la estación. Las Ta-s registradas en las plazas llegan a ser entre $0,5^{\circ} \mathrm{C}$ y $1,5^{\circ} \mathrm{C}$ (registradas a la sombra alcanzan los $4,5^{\circ} \mathrm{C}$ al sol) superiores a las de la estación meteorológica más cercana. Las diferencias en el registro de la HR son aún mayores, llegando hasta el $21 \%$.

Cuando en los estudios bioclimáticos se trabaja con datos históricos medios mensuales ${ }^{7}$ para establecer las estrategias de acondicionamiento pasivo y activo tanto a nivel de edificio como urbano, se observa que la diferencia entre las temperaturas registradas insitu y las temperaturas medias mensuales históricas es aún mayor. Cuando se trabaja con archivos digitales que disponen de datos horarios, los datos han resultado estar más cercanos a la realidad climática local, aunque en todos los casos los datos registrados han sido superiores a los datos de los archivos horarios. En estudios recientes se ha comprobado que estas diferencias de información entre las bases climáticas y los datos reales son las que mayor importancia tienen a la hora de determinar las diferencias entre datos simulados y datos reales, frente a la caracterización de materiales o perfil de uso del espacio (Cuerda, Guerra, Neila \& Sendra 2019).

Las nuevas investigaciones en eficiencia energética y confort higrotérmico están incorporando los efectos de la ICU en la simulación energética (Santamouris, 2014; Bouyer, Inard \& Musy, 2011), aunque en el espacio público a la hora de definir las estrategias para mejorar el confort higrotérmico, se deberá tener en cuenta que las horas de uso son principalmente las diurnas y primeras horas tras el anochecer.

Se ha accedido a los datos de una estación meteorológica privada, ICOMUNID56 Chamberi $i^{8}$, situada en la misma plaza con registros de temperatura, humedad y viento cada 15 minutos. Se han comparado estas mediciones con las registradas en la estación meteorológica estatal de Madrid Retiro. Al comparar las mediciones, se observa el efecto de ICU en la Plaza Chamberí, localizada en un entorno totalmente urbanizado, mientras que la estación de la AEMET se encuentra en el gran parque urbano de El Retiro. Esta puede ser también la causa del registro de Ta superiores en la plaza en las mediciones realizadas in-situ, dado que las curvas de temperatura son muy similares, pero tienen un desfase temporal.

6 Datos extraídos de AEMET para el periodo de mediciones de este estudio. Estación Madrid Retiro. URL: https://opendata.aemet.es/ centrodedescargas/productosAEMET?

\section{$7 \quad$ Archivos climáticos .MET del Código}

Técnico de la Edificación. URL: https:/l www.codigotecnico.org/index.php/menudocumentoscte/133-ct-documentos-cte/ahorro-deenergia.html y Energy Plus Weather Data .EPW. URL: https://energyplus.net/weather.

$8 \quad$ URL: http://www.wunderground. com/weatherstation/WXDailyHistory. asp?ID=ICOMUNID56 


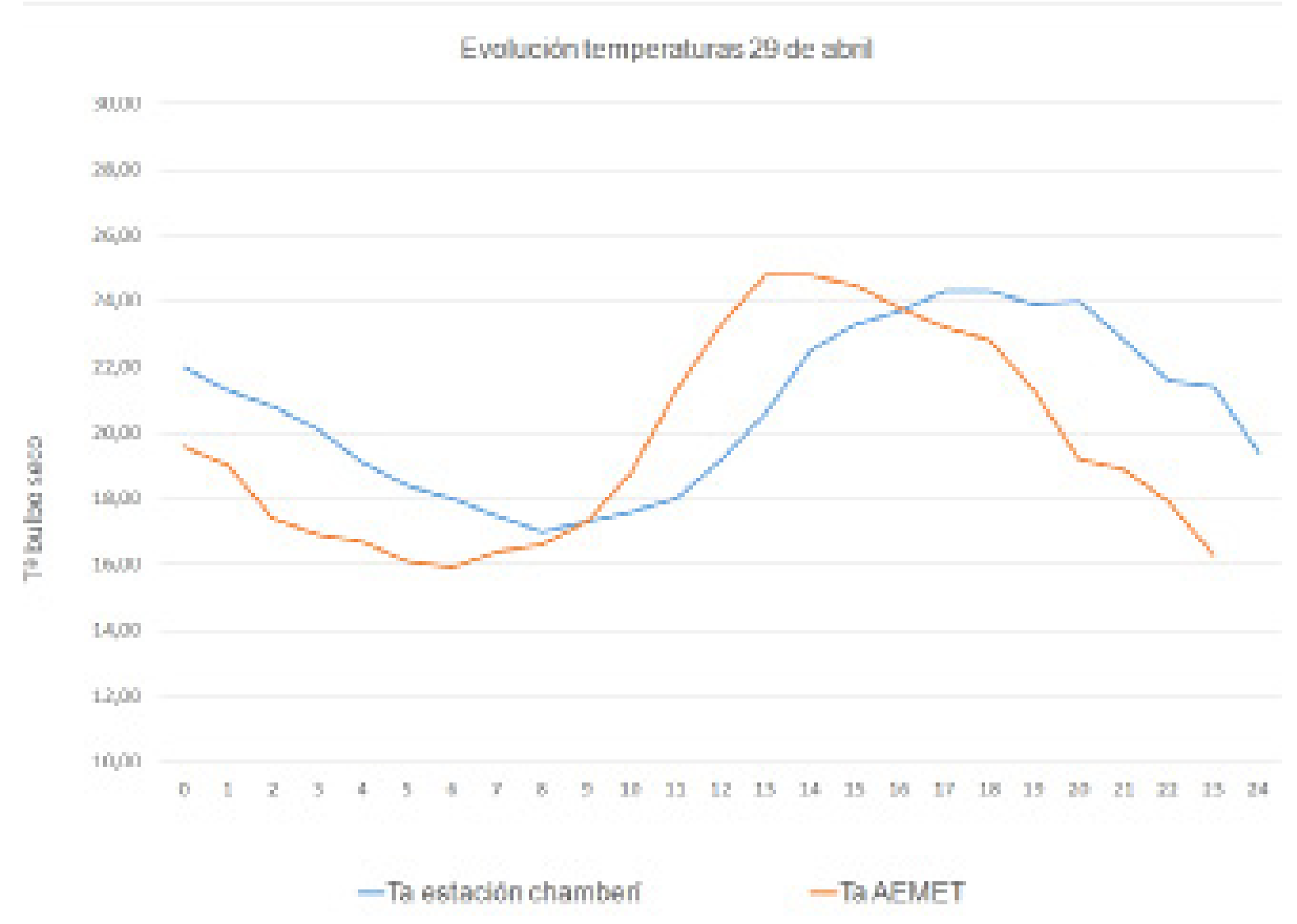

Figura 11. Comparativa de temperatura de bulbo seco horaria registrada por la AEMET en Parque del Retiro y la registrada en una estación meteorológica privada localizada en la plaza Chamberí. Se distingue claramente el efecto de ICU. Fuente: Elaboración propia.
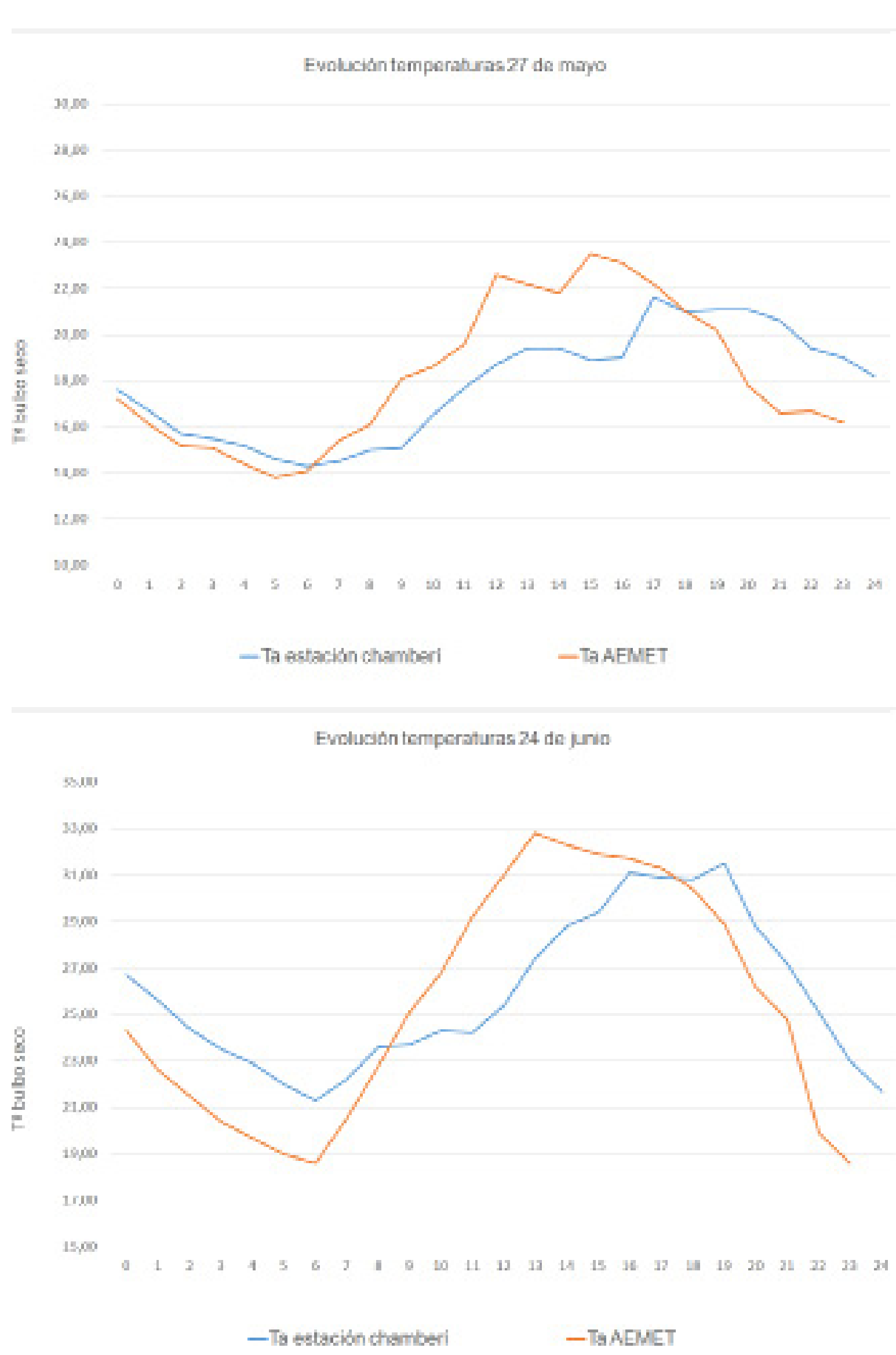
Sobre las mediciones ambientales y el comportamiento térmico de los materiales

Del análisis realizado, se puede observar que el clima madrileño en verano es extremadamente cálido, incluso en horas nocturnas (10:00 p.m.), cuando se midieron $31,3^{\circ} \mathrm{C}$ de Ta, y seco, pues la humedad relativa se encuentra por debajo del $30 \%$ en numerosos momentos a lo largo del día. Por otra parte, se hace patente la reducción de la Ta y el incremento de la HR en los espacios sombreados y en los espacios cercanos al agua, aunque cuanto mayor es la temperatura exterior, su efecto es menor. Lo mismo ocurre en los espacios con suelo de arena y rodeados de arbolado, aunque es un parámetro dependiente de las condiciones climáticas (Fabbri, Canuti \& Ugolini, 2017). La velocidad del viento no supera los $405 \mathrm{~m} / \mathrm{s}$, por lo que no tiene efectos mecánicos.

Los materiales de acabado influyen tanto en el balance térmico como en el balance hídrico (Lee \& French, 2009) del espacio urbano. Sus propiedades térmicas, hídricas y ópticas, principalmente el albedo, absortividad, inercia térmica, permeabilidad al agua y textura, influyen en el balance energético con su entorno, creando condiciones microclimáticas diferenciadas (Fariña et al., 2013; Santamouris, 2001).

En los momentos más cálidos del día, se hacen patentes las diferencias en el comportamiento térmico de los materiales, dándose grandes diferencias de temperatura superficial entre ellos. Se han identificado que esas diferencias en el comportamiento térmico de los materiales están ligadas al albedo, la inercia térmica, densidad y el sombreamiento, de las que el albedo es una propiedad determinante. Se ha comprobado, como numerosos estudios afirman que los materiales de pavimentación con alto albedo mejoran el ambiente térmico urbano en momentos calurosos en combinación con un alto Sky View Factor, reduciendo su temperatura superficial, la temperatura del aire cercano a las superficies y la radiación de onda larga emitida por estas (Dimoudi et al., 2014; Synnefa et al., 2011). En fachadas, aquellas con un mayor albedo también mantienen temperaturas superficiales inferiores.

En junio, el mes más caluroso del periodo del estudio (y prácticamente de todo el año, dado que la medición es del 24 de junio y el momento más caluroso en Madrid es a comienzos de julio), se hace notable la diferencia en el comportamiento térmico entre los materiales menos densos y más densos. Éstos ganan calor más lentamente, pero también se enfrían más lentamente, mientras que aquellos lo hacen más rápidamente alcanzando temperaturas inferiores en la noche.

Los materiales tienen un comportamiento similar en su evolución de temperaturas a lo largo del día, tanto al sol como a la sombra, registrándose TC-s notablemente inferiores a la sombra. Las diferencias de TC entre los mismos materiales al sol y a la sombra llega a ser de hasta $12^{\circ} \mathrm{C}$. El sombreamiento resulta ser una estrategia muy efectiva para la mejora del confort en los momentos más cálidos.

Las altas TC de los materiales convierten la plaza en un lugar con una sensación de calor percibida aún mayor debido al intercambio radiante que habrá entre los acabados y las personas próximas, siendo fuentes de calor a partir del mes de mayo. Teniendo en cuenta los resultados obtenidos del análisis, se hace patente la importancia de la adecuación del diseño a las condiciones climáticas reales en un clima como el madrileño, donde será apropiado el uso de materiales con una baja inercia térmica en aquellas zonas continuamente soleadas durante los meses más cálidos, mientras que los materiales con mayor capacidad de acumulación de energía podrán ser empleados en las zonas soleadas de otoño, invierno y primavera. 


\section{Sobre las condiciones microclimáticas y el uso de la plaza}

Respecto al uso que hacen las personas del espacio, según Gehl (2001) el 96\% de las actividades que se dan en la calle ocurren en el borde público-privado; sin embargo, el perímetro de esta plaza, al tener carácter de paso, existir terrazas privadas y viales cercanos con gran afluencia de coches, ese borde es escasamente utilizado.

También hay un espacio central entre la plaza y las zonas cercanas al quiosco que se ha mantenido sin uso en los días en los que se ha realizado el estudio. Esta zona central de la plaza es donde más horas de sol se reciben durante los meses de primavera, más de 1000 horas, y en este punto no existe arbolado u otros elementos que permitan la protección frente al sol. Es el punto con mayor radiación acumulada, 489600Wh al año, y mayor radiación media por hora, con más de $428 \mathrm{Wh}$. Sin embargo, este espacio tampoco se emplea durante la noche. El calor acumulado en los pavimentos y el efecto de la ICU puede influir en este hecho. Tampoco existen referencias o mobiliario urbano, pudiendo ser ésta otra causa de su falta de uso, dado que se ha observado que la gente se tiende a agrupar en torno a los bancos o mobiliario urbano que sirva de referencia.

Figura 12. Registro gráfico del uso de la plaza. En rojo se señalan las zonas de las que no se hizo uso en los días estudiados. Fuente: Elaboración propia.
Durante las horas de sol, estos son los espacios más empleados. Por la noche el espacio se ocupa de forma diversa y, aunque el diseño tenga influencia, la ocupación del espacio es más flexible.

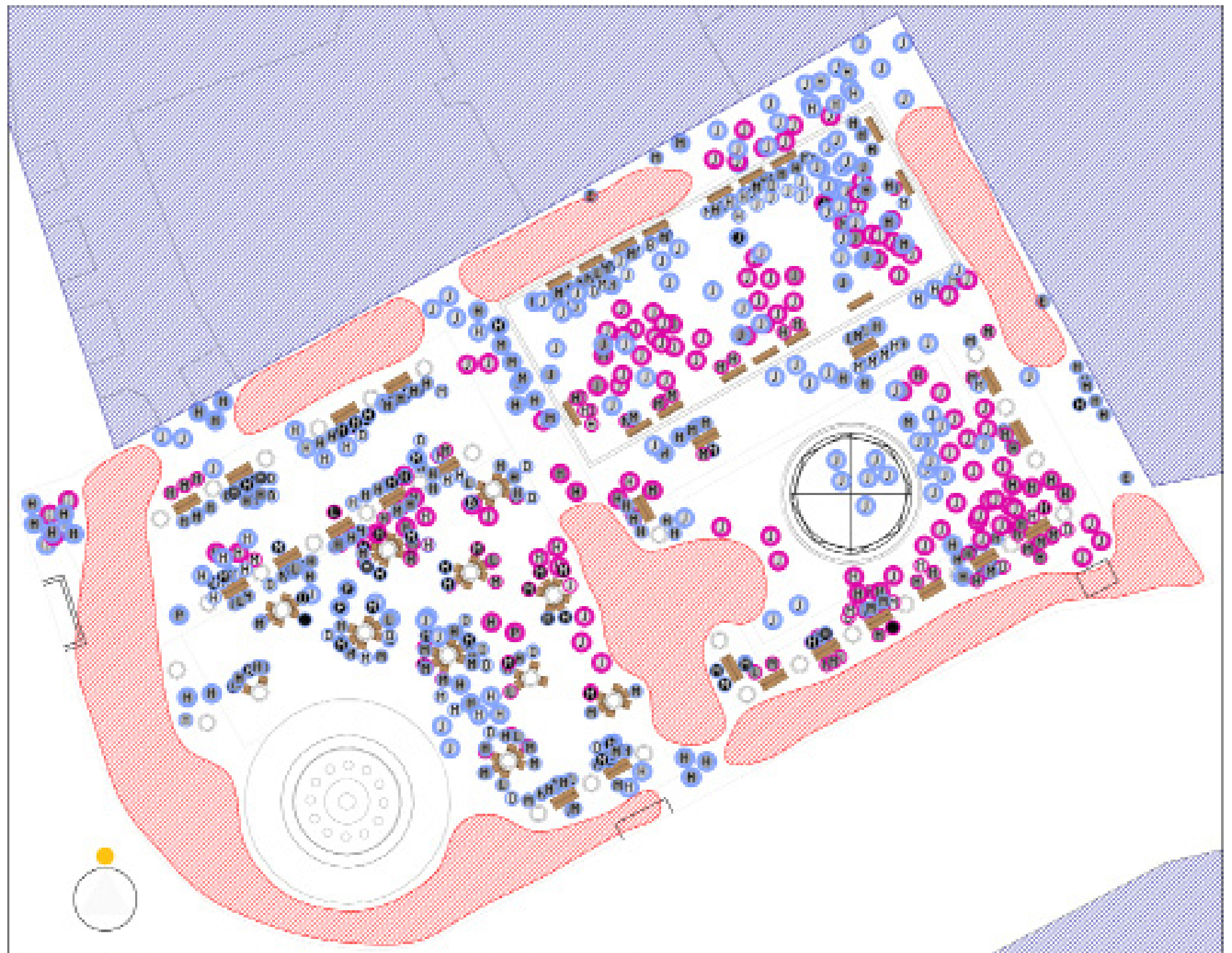

LOCALIZALIUN DE PEASLHAS: PLAZA LEL 2 DE MAYO prinamera.

\begin{tabular}{|c|c|c|c|c|}
\hline \multicolumn{2}{|c|}{ Hünero de personas } & niñss & Alultos & Antifrus: \\
\hline Totsil & ex nuxi & NH ISLAN & J4! & Q1 (TLS) \\
\hline
\end{tabular}

ACTMICADES PAIRIIPALES: PLAZA DEL 2 LE HAYO arindura 
Existen puntos en los que la gente se sitúa reiteradamente a esperar: esquinas y puntos con buena visibilidad. Son también zonas donde la gente permanece en pie hablando por encuentros casuales. Durante los meses analizados, las esperas se han realizado principalmente a la sombra.

Los niños son los únicos que emplean todo el espacio en su conjunto y ocupan aquellas zonas que los adultos no utilizan, como el perímetro de la plaza o el quiosco a diferente nivel, siendo ellos los que rompen las teorías existentes (Gehl, 2001) respecto a la ocupación y uso del espacio público.

Respecto a la relación entre el uso del espacio y las condiciones microclimáticas, las personas pueden realizar diversas acciones ligadas a la localización en el espacio para acondicionarse al entorno climático en búsqueda del confort higrotérmico:

a. Empleo del espacio en horas de más o menos calor

b. Situarse al sol o a la sombra

c. Estar cerca de vegetación

d. En pavimentos de alta o baja inercia térmica y con mayor o menor albedo

e. En cercanía o no a fachadas que irradien calor

f. Sentados en bancos con baja o alta inercia térmica

Chamberí es una plaza con mucha exposición al sol, pero el arbolado existente crea zonas de sombra. En los días analizados de abril, mayo y junio se encontraron más personas a la sombra, 318, que al sol, 218. En las zonas al sol más calurosas y sin equipamientos o bancos, es difícil encontrar personas realizando algún tipo de actividad estancial. Lo mismo ocurre con aquellas zonas a la sombra menos frescas y sin equipamientos o mobiliario urbano. Los espacios a la sombra más empleados son los que tienen un carácter más fresco por disponer de pavimentos ligeros y arbolado. En junio se contabilizaron tan solo 45 personas por la tarde, mientras que por la noche la cifra incrementó a 83 personas. Durante junio, la plaza fue más empleada por la noche.

Se observa que desde abril hasta junio se produce una reducción en el número de personas que emplean la plaza. En abril, el espacio se emplea de un modo más homogéneo, mientras que en el mes de junio se observa una localización más concreta de las personas en torno a las zonas arboladas, los bancos y el área de juegos de niños, todos ellos puntos en los que se dispone de sombra. Se puede observar la relación directa entre el endurecimiento de las condiciones climáticas y el uso del espacio. Esta dinámica se ha confirmado en investigaciones más desarrolladas que la actual (Nikolopoulou \& Lykoudis, 2007).

Según los climogramas desarrollados a lo largo de abril, mayo y en las mañanas de junio, se estará en confort en espacios soleados. Sin embargo, durante los días analizados, en las mañanas de abril y mayo, las personas realizaban sus actividades al sol, pero por las tardes de estos meses había más gente situada a la sombra. En junio, desde la mañana, más del $70 \%$ de la gente se localizaba a la sombra.

En abril no existen grandes diferencias en número entre la gente al sol (43.2\%) y a la sombra $(56.8 \%)$, pero esa diferencia va incrementando notablemente durante mayo con un $61.1 \%$ de la gente a la sombra y durante junio con un $77 \%$. Este comportamiento se hace patente en las personas de todas las edades, aunque es especialmente claro en los adultos y en los ancianos. Los niños, aunque también tienden a estar más al sol, según avanzan los meses de primavera se sitúan mucho más a menudo a la sombra.

Una vez comparada la utilización del espacio que ha hecho la gente en los días analizados y los resultados obtenidos del estudio climático cabe concluir que, en numerosas ocasiones, las estrategias recomendadas por el estudio climático para alcanzar el bienestar no coinciden con el uso real que hace la gente del espacio de las plazas.

Los niños tampoco se adecúan a los resultados de los estudios de confort higrotérmico tradicionales, ya que tienen unos márgenes más amplios de confort, soportando el calor con mayor facilidad aún y cuando realizan actividades de mayor desgaste. Aun 
así, durante el mes de junio prácticamente no juegan al sol por la tarde, hecho que no sucede en los meses anteriores.

Cabe señalar que además del estudio climático teórico con las bases climáticas con medias de 30 años de la AEMET y con las bases climáticas de Energy Plus y del Código Técnico de la Edificación, se han tomado las mediciones realizadas in-situ y se han trasladado a las tablas de las temperaturas horarias. En ese caso, las estrategias recomendadas por el estudio climático correspondientes a esas temperaturas reales coinciden en mayor medida con el uso que la gente ha hecho del espacio, aunque no en todas las ocasiones.

\section{Conclusiones}

Este trabajo analiza la relación entre los análisis climáticos teóricos, las mediciones locales de las variables climáticas y el uso que hacen las personas de la plaza Chamberí de Madrid en los meses de abril a junio. También se comparan los resultados obtenidos con las bases climáticas existentes. Se define la importancia de los materiales, la vegetación, el agua y el sombreamiento en la creación de espacios con diversos matices climáticos y con riqueza microclimática de cara a obtener un mayor confort para las personas. Se identifica la ICU existente entre el Parque del Retiro y la Plaza Chamberí.

Se hace imprescindible un acercamiento al diseño urbano que contemple las variables climáticas para la creación de espacios confortables y vivos que fomenten las relaciones sociales. Las herramientas bioclimáticas son eficaces para la toma de decisiones de un diseño del espacio público que tenga en cuenta las necesidades de las personas y para el diseño y localización de pavimentos, vegetación y espacios estanciales (Gaitani et al., 2014), evitando situaciones no deseables o espacios infrautilizados.

La elaboración de climogramas de bienestar adaptado, aún y cuando toman en cuenta parámetros como la actividad metabólica que se está desarrollando o el arropamiento, no resultan estrictamente empleables para definir el confort o el comportamiento que la gente tendrá en el uso del espacio público. Tal y como se ha venido investigando en los últimos 20 años, resulta imprescindible tener en cuenta parámetros de adaptación fisiológica, física y psicológica (Nikolopoulou, Baker \& Steemers, 1999). El uso que hacen los niños del espacio se aleja aún más de las estrategias de confort que señalan estos estudios. El confort higrotérmico se basará en la creación de espacios microclimáticos variados, estando fuertemente condicionado por las opciones de ocupación del espacio que existan y que permitan la adaptación al medio. Para ello, desde el diseño urbano, se requiere de la combinación de suelos naturales y materiales con diversas propiedades higrotérmicas, principalmente en lo referente a capacidad de almacenaje de energía solar, reflectancia solar y permeabilidad. Al mismo tiempo, en climas tan variables como el madrileño, es imprescindible la realización de análisis de soleamiento-sombreamiento en la época de frío y en la de calor para la localización de los suelos naturales, la selección de tipos de materiales, su cantidad y el empleo de agua y vegetación. Todo ello permitirá la creación de una variedad de microclimas para optar por soluciones que reduzcan el efecto de ICU.

La dificultad de caracterización climática de los espacios exteriores urbanos reside en que las condiciones microclimáticas de estos espacios son difícilmente generalizables, careciendo en muchas ocasiones de datos climáticos en puntos cercanos a la zona de estudio (Rogora \& Dessí, 2005). Además, las temperaturas medias de estaciones meteorológicas con las que se trabaja en el diseño bioclimático incluyen las horas más frías de las noches, momentos en los que, en general, los espacios públicos no están en uso. Finalmente, como se ha visto en este estudio, los datos climáticos empleados no incluyen la ICU que sufren muchos de los espacios urbanos consolidados y que deben ser diseñados o remodelados.

Las conclusiones aquí extraídas sobre el comportamiento y uso que hace la gente de las plazas no son generalizables, pues tan sólo corresponden a los días en los que se han llevado a cabo los trabajos de campo. Sin embargo, los trabajos de observación y de medición realizadas en las plazas han puesto de manifiesto lo variable del clima y como 
ante la necesidad de trabajar con unos valores generales de temperaturas y humedades medias, en ocasiones, la realidad del lugar no coincide con esa generalización.

En el espacio público, expuestos al clima exterior que es estacional y variable, se trabaja con parámetros difícilmente controlables a la hora de querer diseñar lugares confortables. Además, estos espacios deben ser adecuados a diferentes personas con diferentes percepciones del confort, realizando diferentes actividades y con diferentes intensidades metabólicas. Así, el objetivo del diseñador urbano que quiere crear espacios urbanos confortables debe ser ofrecer un espacio con variadas situaciones microclimáticas, pudiendo así cubrir las actividades que los ciudadanos desean 0 necesitan realizar a lo largo de todo el año.

\section{Agradecimientos}

Los autores agradecen la cesión gratuita de los datos climáticos requeridos para la investigación a la Agencia Estatal de Meteorología AEMET.

\section{Referencias bibliográficas}

Bouyer, J., Inard, C. y Musy, M. (2011). Microclimatic coupling as a solution to improve building energy simulation in an urban context. Energy and Buildings, 43(7), 15491559.

Brown, R.D. (2011). Ameliorating the effects of climate change: Modifying microclimates through design. Landscape and Urban Planning; 100, 372-74.

Chen, L. y Ng E. (2012). Outdoor thermal comfort and outdoor activities: A review of research in the past decade. Cities, 29, 118-125.

Coutts, A.M. y Harris, R. (2013). A multi-scale assessment of urban heating in Melbourne during an extreme heat event and policy approaches for adaptation. Technical Report, p. 64. Melbourne: Victoria Centre for Climate Change and Adaptation Research.

Cuerda, E., Guerra, O., Neila, F.J. y Sendra J. (2019). Comparing the impact of occupancy patterns on energy demand in residential buildings using measured data and simulation models. Building Simulation, 1-14.

Dimoudi, A., Zoras, S., Kantzioura, A., Stogiannou, X., Kosmopoulos, P. y Pallas C. (2014). Use of cool materials and other bioclimatic interventions in outdoor places in order to mitigate the urban heat island in a medium size city in Greece. Sustainable Cities and Society, 13, 89-96.

Erell, E., Pearlmutter, D. y Williamson, T. (2010). Urban microclimate: designing the spaces between buildings. London: Earthscan.

European Commission (2017). Two years after Paris: Progress towards meeting the EU's climate commitments. Report: COM(2017) 646 final. Brussels.

European Environment Agency (2012). Urban adaptation to climate change in Europe. Challenges and opportunities for cities together with supportive national and European policies. EEA Report No 2/2012, 148.

Fabbri, K., Canuti, G. y Ugolini, A.(2017). A methodology to evaluate outdoor microclimate of the archaeological site and vegetation role: A case study of the Roman Villa in Russi (Italy). Sustainable Cities and Society, 35, 107-133.

Fanger, P. O. (1982). Thermal comfort. Analysis and application in environment engineering. New York: McGraw Hill Book Company.

Fariña, J., Fernández, V., Galvez, M.A., Hernández, A. y Urrutia, N. (2013). Manual de diseño bioclimático urbano. Recomendaciones para la elaboración de normativas urbanísticas. Bragança: Instituto Politécnico de Bragança. 
Fernández F., Allende F., Rasilla D., Martilli A. y Alcaide J. (2016). Estudio de detalle del clima urbano de Madrid. Área de Gobierno de Medio Ambiente y Movilidad. Madrid: Ayuntamiento de Madrid.

Gaitani, N., Mihalakakou, G. y Santamouris, M. (2007). On the use of bioclimatic architecture principles in order to improve thermal comfort conditions in outdoor spaces. Build Environment, 42, 317-324.

Gaitani, N., Santamouris, M., Cartalis, C., Pappas, I., Xyrafi, F., Mastrapostoli, E., Karahaliou, P. y Efthymiou, Ch. (2014). Microclimatic analysis as a prerequisite for sustainable urbanisation: Application for an urban regeneration project for a medium size city in the greater urban agglomeration of Athens, Greece. Sustainable Cities and Society, 13, 230-236.

Gehl, J. (2001). Life between buildings. Using Public Space. Copenague: Copenhagen Arkitektens Forlag. The Danish Architectural Press.

Givoni, B. (1998). Climate considerations in building and urban design. New York: John Wiley and sons.

Givoni, B., Noguchi, M., Saaroni, H., Pochter, O., Yaacov, Y., Feller, N. y Becker, S. (2003). Outdoor comfort research issues. Energy and Buildings, 35(1), 77-86.

Howard, L. (1883). The Climate of London; Volumen I-III. London: Harvey and Dorton.

Hui, L. (2015). Pavement Materials for Heat Island Mitigation: . Design and Management Strategies. Oxford: Butterworth-Heinemann.

IPCC (2007). En: Soloman, S., Qin, D., Manning, M., Chen, Z., Marquis, M., Averyt, K.B., Tignor, M. y Miller, HL. (eds) Climate change 2007: the physical science basis. Contribution of working group I to the fourth assessment report of the intergovernmental panel on climate change. Cambridge: Cambridge University Press.

Landsberg, H. (1981). The Urban Climate. London: Academic Press.

Lee, SH. y French, SP. (2009). Regional impervious surface estimation: an urban heat island application. Journal of Environmental Planning and Management; 52(4), 477-96.

Lenzholzer, S. (2010). Engrained experience: a comparison of microclimate perception schemata and microclimate measurements in Dutch urban squares. International Journal of Biometeorology, 54(2), 141-150.

Luber, G. y McGeehin, M. (2008). Climate Change and Extreme Heat Events. American Journal of Preventive Medicine. 35(5), 429-435.

Mayer, H. y Höppe, P. (1987). Thermal comfort of man in different urban environments. Theoretical and Applied Climatology, 38, 43-49.

Ministerio de Fomento (2010). Catálogo de elementos constructivos del Código Técnico de la Edificación. Madrid: Gobierno de España. URL: https://itec.cat/cecl

Neila, J. (2004). Arquitectura bioclimática en un entorno sostenible. Madrid: MunillaLería.

Nikolopoulou, M., Baker N. y Steemers K. (1999). Thermal comfort in urban spaces: different forms of adaptation. En REBUILD International Conference: The Cities of Tomorrow. Barcelona.

Nikolopoulou, M. y Lykoudis, S. (2006). Thermal comfort in outdoor urban spaces: understanding the human parameter. Solar Energy, 70(3), 227-235.

Nikolopoulou, M. y Lykoudis S. (2007). Use of outdoor spaces and microclimate in a Mediterranean urban area. Building and Environment, 42(10), 3691-3707.

Núñez Peiró, M., Sánchez-Guevara Sánchez, C. y Neila González, F.J. (2017). Actualización de la isla de calor urbana de Madrid y su influencia en la simulación energética de edificios. En Proceedings of the 3rd International Congress on Sustainable Construction and Eco-Efficient Solutions, 890-901, Sevilla: Universidad de Sevilla. 
Oke, T.R. (1973). City size and the urban heat island. Atmospheric Environment 7, 769779.

Oke, T.R. (1982). The Energetic Basis of the Urban Heat Island. Quarterly Journal of the Royal Meteorological Society, 108, 1-24.

Olgyay, V. (1999). Design with climate: Bioclimatic Approach toArchitectural Regionalism. New Jersey: Princeton University Press, Princeton, 1963. Reed. Arquitectura y clima. Manual de diseño bioclimático para arquitectos y urbanistas. Barcelona: GG.

Oregi, X., Roth, E., Alsema, E., van Ginkel, M. y Struik, D. (2015). Use of ICT tools for integration of energy in urban planning projects. En Sustainability in Energy and Buildings: Proceedings of the 7th International Conference SEB-15. Energy Procedia, 83, 157-166.

Owen. T.W., Carlson, T.N. and Gillies, R.R. (1998). An assessment of satellite remotely - sensed land cover parameters in quantitatively describing the climatic effect of urbanization. International Journal of Remote Sensing,19(9), 1663-1681.

Pearlmutter, D., Jiao, D. y Garb, Y. (2014). The relationship between bioclimatic thermal stress and subjective thermal sensation in pedestrian spaces. International Journal of Biometeorology, 58(10), 2111-2127.

Population Reference Bureau (2009). 2009 World population data sheet. URL: http:// www.prb.org/Publications/Datasheets/2009/2009wpds.aspx>

Rogora, A. y Dessí, V. (2005). II comfort ambientale negli spazi aperti. Gorizia: EdicomEdizioni.

RUROS Project (2004). Designing open spaces in the urban environment: A bioclimatic approach. [online]. Coordinado por CRES (Centre of Renewable Energy Resources). URL: <http:// www.cres.gr/kape/education/1.design_guidelines_ en.pdf>.

Santamouris, M. (2001). Energy and Climate in the Urban Built Environment. Londres: James \& James Science Publishers.

Santamouris, M. (2014). On the energy impact of urban heat island and global warming on buildings. Energy and Buildings, 82, 100-113.

Smargiassi, A., Goldbert, M.S., Plante, C., Fournier, M., Baudouin, Y. y Kosatsky, T. (2009). Variation of daily warm season mortality as a function of micro-urban heat islands. Journal of Epidemiology and Community Health, 63, 659-664.

Shooshtarian, S., Rajagopalan, P. y Sagoo, A. (2018). A comprehensive review of thermal adaptative strategies in outdoor spaces. Sustainable Cities and Society 41, 647-665.

Smid, M., Russo, A., Costa, A.C., Granell, C. y Pebesma, A. (2019). Ranking European capitals by exposure to heat waves and cold waves. Urban Climate 27, 388-402.

Synnefa, A., Karlessi, T., Gaitani, N., Santamouris, M., Assimakopoulos, DN. y Papakatsikas, C. (2011). Experimental testing of cool colored thin layer asphalt and estimation of its potential to improve the urban microclimate. Building and Environment, 46(1), 38-44.

Whyte, W.H. (1980). The social life of small urban spaces. Washington, D.C. : Project for public spaces. 